\title{
Clinical parameters and biomarkers predicting spontaneous operational tolerance after liver transplantation: a scoping review
}

Christian Appenzeller-Herzog ${ }^{1}$, Steffen Hartleif ${ }^{2}$, and Julien Vionnet ${ }^{3-5}$

Affiliations:

(1) University Medical Library, University of Basel, Spiegelgasse 5, 4051 Basel, Switzerland, ORCID https://orcid.org/0000-0001-7430-294X

(2) University Hospital Tübingen, Pediatric Gastroenterology and Hepatology, Hoppe-Seyler-Str. 1, 70597 Stuttgart, Germany, , ORCID https://orcid.org/0000-0002-2608-5087

(3) Institute of Liver Studies, King's College Hospital, London, United Kingdom, ORCID https://orcid.org/00000002-1654-0488

(4) Transplantation Centre, University Hospital of Lausanne and University of Lausanne, Lausanne, Switzerland

(5) Service of Gastroenterology and Hepatology, University Hospital of Lausanne and University of Lausanne, Lausanne, Switzerland

Corresponding author: Christian Appenzeller-Herzog, Christian.Appenzeller@unibas.ch

Abbreviations:

APC, antigen-presenting cell; CNI, calcineurin inhibitors; CENTRAL, Cochrane Central Register of Controlled Trials; DC, dendritic cells; DSA, donor specific antibodies; IS, immunosuppression; ISW, immunosuppression withdrawal; LFT, liver function tests; LT, liver transplantation; mTOR-I, mammalian target of rapamycin inhibitor; NINV, non-autoimmune/non-replicative viral; NK, natural killer; OT, operational tolerance; PBMC, peripheral blood mononuclear cells; SOT, solid organ transplantation; Treg, regulatory T cells 


\section{Abstract}

Indefinite allograft acceptance after immunosuppression withdrawal (ISW), also known as operational tolerance (OT), can occur spontaneously after liver transplantation (LT) but reliable and reproducible prognosis of OT versus non-OT outcomes remains elusive. To prime this, systematic extraction of OT-predictive factors from the literature is crucial. We provide the first comprehensive identification and synthesis of clinical parameters and biomarkers predicting spontaneous OT in non-autoimmune/non-replicative viral LT recipients selected for ISW. We searched Embase, Medline, the Cochrane Central Register of Controlled Trials, clinicaltrials.gov, and the World Health Organization International Clinical Trials Registry Platform for articles, conference abstracts, and ongoing trials. We contacted principal investigators of stand-alone abstracts and ongoing trials for unpublished data and screened citations and references of eligible articles. 23 articles reporting on 11 completed ISW studies, 13 abstracts, and five trial registry entries were included.

Longer time between LT and ISW was the only clinical parameter that may increase the incidence of OT. Prognostic biomarkers conspicuously differed between pediatric and adult ISW candidates. These included allograft gene expression patterns and peripheral blood immune exhaustion markers for adults, and histological allograft scores for children. Our results will foster cross-validation efforts to facilitate safe and harmonized candidate selection for successful ISW. 


\section{Introduction}

The introduction of potent pharmacological immunosuppression (IS) regimens in the late 1970s, in particular calcineurin inhibitors (CNI), kick-started the medical and surgical success story of solid-organ transplantation (SOT). While preventing acute and chronic rejection, these treatments are directly or indirectly responsible for various complications including recurrent or de novo malignancies, cardiovascular and metabolic diseases, chronic kidney disease and infections, which jeopardize patient and allograft survival. ${ }^{1-3}$ Achieving drug-free allograft survival in SOT, known as operational tolerance (OT), would allow to overcome these adversities, while preserving a long-term allograft function. ${ }^{4}$

Among all solid-organ transplants, the liver exhibits unique and complex immunoregulatory properties, which render liver allografts less dependent on IS and less sensitive to immunological damage. ${ }^{5}$ The putative underlying mechanisms of tolerance may include large antigen load, deficient antigen presentation by antigen-presenting cells (APC), neutralization of alloantibodies, deletion of effector lymphocyte clones, regulatory T cell (Treg) generation as well as long-term microchimerism. ${ }^{6,7}$ Based on these particular features, clinical studies that examined IS minimization or complete IS withdrawal (ISW) following pediatric and adult liver transplantation (LT) have been initiated in the $1990 \mathrm{~s}^{8}$ It is now estimated that $20-40 \%$ of carefully selected nonautoimmune/non-replicative viral (NINV) LT recipients can eventually achieve OT by stopping IS and yet maintaining a stable allograft function and histological integrity. ${ }^{7}$ The majority of LT recipients would, however, still experience an acute cellular rejection episode or develop abnormal liver function tests (LFT) during the ISW process and require reinstitution of IS.,8 The timeline of the consecutive steps of an ISW study is illustrated in Figure 1.

The discovery of OT has promoted extensive research activity over the last two decades. One studied objective was to explore the factors associated with the development of OT to help refine the eligibility criteria for LT recipients to participate in ISW trials and increase the fraction of successful ISW. ${ }^{9}$ Furthermore, researchers have started to address the question as to whether OT can be induced by immune manipulation prior to ISW. ${ }^{4}$ 
After two decades of OT research, we aim to systematically identify, review, and summarize the evidence on clinical parameters and biomarkers predicting spontaneous OT in prospective ISW LT recipients. The comprehensive mapping will likely foster inter-center cross-validation of OT-predictive factors with the final goal of concerted, safe, and successful ISW candidate selection after LT.

\subsection{Study aim and objectives/questions}

The objective of this scoping review was to map all published prognostic factors for spontaneous OT in NINV LT recipients who are being subjected to ISW. The obtained results may inform the subsequent conduct of a systematic review with a more targeted review question.

Specifically, the review questions were:

i) What are clinical parameters and biomarkers that predispose LT recipient ISW candidates to achieve spontaneous OT?

ii) What are the success rates of ISW and achievement of spontaneous OT in LT recipients?

iii) What are the rates of graft loss in LT recipients following ISW? 


\section{Methods}

\subsection{Protocol and registration}

This scoping review was conducted along with the guidelines by the Joanna Briggs Institute ${ }^{10}$ and reported according to the PRISMA-ScR statement. ${ }^{11}$ A study protocol was prospectively peer-reviewed and published. ${ }^{12}$

\subsection{Eligibility criteria}

\subsubsection{Population, Intervention, Outcomes}

The primary eligibility criterion was the assessment of spontaneous OT, i.e. rejection-free liver allograft survival for at least one year following ISW. LT recipients of any age or stage were included, but recipients with underlying autoimmune diseases, replicative viral disease (i.e. patients with positive hepatitis B and/or C viremia detected by polymerase chain reaction) and/or multi-organ recipients were excluded. Studies reporting on mixed populations were included if NINV study population was $80 \%$ or greater. Studies that did not report the liver disease etiologies for LT in their population were also included. All pharmacological IS regimens, including combination treatments, being completely withdrawn were eligible. However, studies addressing dose reduction of IS including IS minimization, withdrawal of a subset of drugs from IS combination treatments (e.g. withdrawal of corticosteroids in patients on CNI maintenance treatment), or conversion between IS regimens [e.g. CNI to mammalian target of rapamycin inhibitor (mTOR-I) conversion vs. CNI continuation] were excluded.

We exclusively included studies that assessed an association of pre-ISW clinical parameters or pre-ISW biomarkers with OT development. Studies that addressed the effectiveness of induction or immunomodulation therapies on OT development were excluded. Owing to the risk of confounding by interrupted IS in the OT cohort, data on post-ISW biomarkers were excluded unless the same biomarkers had been measured in the same patients already before ISW.

\subsubsection{Study types}

We included prospective, retrospective, randomized, and non-randomized ISW studies irrespective of publication status, including case-control and cross-sectional designs. Conference abstracts and trial registry entries where the data was already published in a peer-reviewed article were excluded. Animal studies, case reports, case series (i.e. publications where patient histories of exclusively OT or non-OT LT recipients are 
reported), reviews, letters, and editorials were excluded. No language or publication date restrictions were applied.

\subsection{Information sources}

An information specialist (CA-H) developed the search strategies, which were reviewed by a second information specialist (HE). Database-specific subject headings and text word synonyms for LT, ISW, OT, graft survival, or liver biopsy were used. We searched Embase via Elsevier, Medline via Ovid, and the Cochrane Central Register of Controlled Trials (CENTRAL) (last search July 17, 2019; Supplementary Information). We also searched the study registry clinicaltrials.gov as well as the World Health Organization's International Clinical Trials Registry Platform for ongoing studies (last search September 3, 2019; Appendix 1). All retrieved references were exported to EndNote X9 and deduplicated.

\subsection{Selection of sources of evidence}

One reviewer $(\mathrm{CA}-\mathrm{H})$ screened the references based on their titles and abstracts. All potentially relevant references were retrieved in full-text and independently assessed by two reviewers (CA-H, JV). Any disagreements over eligibility were resolved by consensus. Where necessary, a third review author (SH) made a final judgment.

To identify possible additional studies that escaped our database searches, we screened the references and citations in Scopus of all included articles (April 30, 2020). Furthermore, principal investigators of identified relevant ongoing studies and conference abstracts were contacted twice by email for the sharing of any unpublished data (personal communications, preprints, manuscripts in press).

\subsection{Data charting process}

Two reviewers (CA-H, JV) independently charted the data from each source of evidence using a jointly developed MS Excel 2016 charting form that was pilot-tested with four eligible full-text articles. The charting form was updated in an iterative process. Any disagreements over charted data items were solved by discussion. 


\subsection{Data items}

Next to reported prognostic and non-prognostic factors (clinical parameters and biomarkers) for OT, which were the primary outcomes, we charted the incidence of OT and the number of graft losses in each trial as the secondary outcomes. All charted data items are listed in Table S1.

\subsection{Synthesis of results}

For the synthesis of prognostic clinical parameters and biomarkers, we used descriptive statistics to highlight sources of evidence that supported or invalidated each factor. For the calculation of the pooled incidence of OT from completed ISW studies, the reports with the highest number of study participants were chosen in case of multiple reports of the same study. In addition to tabular views, the results were narratively synthesized. 


\section{Results}

\subsection{Results of the search and study characteristics}

Our bibliographic database searches returned 4,704 unique hits, which included 1,185 Embase- or CENTRALderived conference abstracts. Our clinical trial registry searches returned 87 unique hits and 20 additional potentially relevant articles were derived iteratively from registry entries or conference abstracts, or found by citation tracking of included articles, or by contact with study authors. Out of the 193 selected full-text records, 152 did not meet our inclusion criteria for the reasons indicated (Figure 2; Table S2). The remaining 23 articles ${ }^{13-}$ ${ }^{35}$ (Table S3), 13 conference abstracts ${ }^{36-48}$ (Table S4), and five registry entries ${ }^{49-53}$ (Table S5) were included (Figure 2). They were published between 2001 and 2020.

The 23 eligible articles reported on eleven completed ISW studies, as evidenced by cohort overlap (Table S3). Of the eleven completed ISW studies, six (three prospective and three retrospective studies) reported on pediatric and five (all prospective) on adult LT recipient cohorts (Tables S3, S6, S7). Three monocenter pediatric ISW studies were from Kyoto (Japan), 17,18,20,27 Tochigi (Japan), 22,33 and Taipei (Taiwan) ${ }^{23}$ and three multicenter pediatric ISW studies ${ }^{14,31,35}$ from North American liver transplantation units. Four monocenter adult ISW studies were from Chicago (US), ${ }^{34}$ Murcia (Spain) ${ }^{13,16,21,24,29}$ [two studies], and Pamplona (Spain) ${ }^{26,28}$ [one report $^{32}$ integrated the clinical parameters from one Pamplona and three Murcia trials], and one multicenter adult ISW study $^{15,19,25,30}$ analyzed trial data from Barcelona (Spain), Rome (Italy), and Leuven (Belgium). Population characteristics of ISW study reports including living versus deceased donors and inclusion of patients with viral liver disease etiology or non-elective ISW are detailed in Table S3.

Of the 13 included conference abstracts, two ${ }^{45,47}$ provided additional data derived from one of the completed ISW studies (WISP-R ${ }^{14}$ ), six ${ }^{37-41,48}$ reported on work in progress derived from ongoing trials (see below), and five $\mathrm{e}^{36,42-44,46}$ added potentially new ISW data, which has not been published in an article yet (Table S4). The latter abstracts were from Pittsburgh (US), ${ }^{42,44,46}$ Shanghai (China), ${ }^{36}$ and London (UK). ${ }^{43} 6 / 13$ conference abstracts reported pediatric ${ }^{36,42,44-47}$ and 7/13 adult ${ }^{37-41,43,48}$ ISW research. 
Four of five included trial registry entries related to the ongoing ISW trials ALLTOL (NCTO2743793), ${ }^{51}$ LIFT (NCT02498977), ${ }^{53}$ LITMUS (NCT02541916), ${ }^{52}$ and OPTIMAL (NCT02533180) ${ }^{49}$ and one trial ${ }^{50}$ was stopped due to poor recruitment (A. Sanchez-Fueyo, personal communication 20200709) (Table S5). ALLTOL and OPTIMAL are US multicenter ISW trials, LITMUS is a Canadian monocenter, and LIFT a European multicenter ISW trial, which all have completed their recruitment of adult LT recipients.

\subsection{OT-predicting clinical parameters}

Table 1 summarizes our results on clinical parameters. The most frequently examined prognostic clinical parameter (assessed in 10/11 completed studies) was the time between LT and ISW. Five studies ${ }^{13,14,18-20,25-29,32}$ (two pediatric and three adult) found longer time to ISW to be OT-predictive, four studies ${ }^{16,22,24,34,35}$ (two pediatric and two adult) found no effect (one with an OT-predictive trend), and one pediatric study ${ }^{23}$ indicated an opposite, i.e. non-OT-predictive effect. All other potentially OT-predicting clinical parameters were reported in isolated ISW studies only, whereas for every suggested parameter several other ISW studies that assessed the same parameter found no prognostic value (Table 1, see also Tables S6 and S7 for further clinical parameters from pediatric and adult studies, respectively).

\subsection{OT-predicting biomarkers}

The quality of association of selected pre-ISW biomarkers with OT is summarized in Table 2. Full details of all reported pediatric and adult OT-predicting biomarkers are presented in Tables S6 and S7, respectively.

\subsubsection{Pediatric results}

Published evidence supporting non-invasive OT-predicting biomarkers in children is particularly scarce. Concerning serum biomarkers, one pediatric study found some post-LT but no pre-LT anti-HLA-antibodies to be associated with later OT, ${ }^{22}$ but other studies could not endorse such association even when restricting their analysis to donor-specific antibodies (DSA). ${ }^{14,35}$ Regarding pediatric OT-predicting peripheral blood mononuclear cell (PBMC) markers, none have been reported so far.

Additional non-invasive biomarkers that may predict pediatric OT came from conference abstracts (Table S4, not included in Table 2). Re-analysis of frozen pre-ISW PBMC from the WISP-R study ${ }^{14}$ showed that half of the 
OT samples were characterized by a two-fold increase in exhausted T cells (CD4+ PD-1+ cells), whereas the fraction of these cells in the remaining OT samples was indistinguishable from the one in non-OT samples. ${ }^{45,47}$ The potential importance of PBMC exhaustion markers for pediatric OT-prediction is also supported by other conference abstracts. ${ }^{36,44,46}$

All invasive OT-predicting biomarkers in children were derived from histological analyses. Absence of portal inflammation (but not of lobular or perivenular inflammation) predicted OT in two multicenter studies..$^{14,35}$ Further pre-ISW OT-associated histological markers were fewer APC:lymphocyte pairs, lower C4d score, decreased load of leukocytes, and decreased load of infiltrating monocytes/macrophages. ${ }^{14,35}$

\subsubsection{Adult results}

Non-invasive OT-predicting biomarkers in adults are subdivided into immunoassays, PBMC subsets, PBMC gene expression patterns, and serum markers. Quite prominently among the former, in vitro stimulated proliferation was substantially lower for pre-ISW lymphocytes that were derived from prospective OT compared to non-OT patients. $^{26}$

Two major studies suggested OT-predicting PBMC subset frequencies: a higher fraction of natural killer (NK) cells and a lower fraction of V $\delta 2-\mathrm{TCR} \gamma \delta \mathrm{T}$ cells in the Barcelona/Rome/Leuven study ${ }^{25}$ (the NK result was endorsed with borderline statistics by the Pamplona study ${ }^{26}$ but not supported by two other studies; ${ }^{24,34}$ and the $\gamma \delta$ T result was not endorsed by subsequent work ${ }^{29}$ ) and higher fractions of tolerogenic dendritic cells (DC) or of TEMRA+, and Eomes+ CD8+ T cells accompanied by a lower fraction of naive CD8+ T cells in the Chicago study. ${ }^{34}$ Of note, a higher fraction of Treg, a T cell subpopulation known to be crucial for achieving tolerance, ${ }^{20,25,27,54-56}$ does not appear to have any pre-ISW prognostic value for OT..$^{24-26,29,34}$

Regarding PBMC gene expression markers, a study from Murcia reported OT-predicting expression of FEM1C, SENP6, and the miRNAs miR-95 and miR-31. ${ }^{29}$ In addition, the Barcelona/Rome/Leuven study showed the two pre-ISW gene expression signatures [NCR1, PDGFRB, PSMD14] and [SLAMF7, KLRF1, CLIC3, PSMD14, ALG8, CX3CR1], ${ }^{25}$ the latter of which being related to NK cell expansion, to be moderately OT-predictive. 
In keeping with the prognostic value of allograft gene expression related to iron metabolism (see below), increased ferritin levels and, somewhat less robustly, hepcidin-25 may represent OT-predictive adult serum biomarkers. This is based on Barcelona/Rome/Leuven data ${ }^{25}$ and non-significant trends reported in a Murcia study. ${ }^{29}$ Notably, although being associated with subclinical allograft injury and fibrosis, ${ }^{57}$ pre-ISW anti-HLA antibodies (including DSA) appear not to have any prognostic value for OT. ${ }^{19,34}$

With regards to invasive pre-ISW biomarkers, robust and reproducible prediction of OT was achieved using gene expression analysis in liver biopsies of adult ISW candidates. Specifically, the Barcelona/Rome/Leuven study identified the iron metabolism-related gene expression signature [CDHR2, MIF, PEBP1, SOCS1, TFRC] (and four additional signatures each comprising more than five genes) as a predictor of OT with a sensitivity of $89 \%$, a specificity of $86 \%$, and an overall error rate of $13 \% .{ }^{25}$ These results were largely replicated in the Chicago study that specifically examined mTOR-I ISW. ${ }^{34}$ OT-predicting histological markers were increased load of CD4+ cells ${ }^{34}$ and higher iron deposition in hepatocytes. ${ }^{25}$

Further invasive biomarkers were extracted from conference abstracts (Table S4). Increased allograft expression of the miRNA miR-193a-3p was reported to denote adult patients that are likely to develop OT, ${ }^{43}$ but this biomarker was later abandoned due to lack of robustness (A. Sanchez-Fueyo, personal communication 20200709). Finally, the prognostic value of allograft and PBMC positivity for an eight gene expression panel (six immunoregulatory genes decreased and two pro-inflammatory genes increased) that was derived from preclinical experiments ${ }^{58}$ is currently under investigation. ${ }^{39,40,48,52}$

\subsection{Incidence of OT and graft loss}

The pooled incidence of OT in the 11 completed ISW studies was calculated with data from six articles on pediatric $^{14,20,23,31,33,35}$ and five articles on adult study populations. ${ }^{13,16,19,26,34}$ The pediatric studies included 371 LT recipients, and a median of $36.5 \%$ (range $29-63$ ) of recipients successfully completed ISW. The adult studies included 174 LT recipients, and a median of $42 \%$ (range $29-63$ ) of recipients successfully completed ISW. It is important to stress that these numbers are neither a systematic result nor a meta-analysis, since all ISW studies that did not report any OT-predictive factors were excluded per protocol (see above and Table S2). Likewise, as 
the study populations of all included ISW studies are highly selected (e.g. with no previous rejections or abnormal LFT), the incidence of OT in the general LT recipient population is expected to be substantially lower. In all included studies, only one graft in a pediatric recipient was lost, ${ }^{22,33}$ confirming that - appropriate ISW eligibility provided - ISW failure is not per se associated with a significantly elevated risk of graft loss. ${ }^{7}$ 


\section{Discussion}

This review provides the first systematic overview of published prognostic factors to inform clinicians' and patients' decisions regarding whether or not to embark on ISW after LT. Since successful ISW has many benefits including cessation of toxicity of IS medication,,$^{1-3}$ improved quality of life, ${ }^{59}$ and cost-effectiveness ${ }^{60}$ but ISW failure can be frustrating and associated with undesirable consequences (yet apparently not with graft loss), the clinical value of secured prognostic information on ISW outcomes is evident. To the best of our knowledge, the rigorous eligibility criteria that guided this review and led to the exclusion of all OT-associated biomarkers manifesting only during or after ISW were unique. Indeed, we could show that according to current evidence, frequently quoted OT biomarkers such as DC2:DC1 ratio, ${ }^{61} \mathrm{HLA}-\mathrm{G}$ level, ${ }^{54,62}$ or V $\delta 1: \mathrm{V} \delta 2 \gamma \delta \mathrm{T}$ cell subset ratio ${ }^{63}$ cannot predict OT before ISW and guide ISW decisions (Table S2). Furthermore, the predictive capacities of most of the pre-ISW clinical parameters and of some biomarkers did not withstand inter-study cross-check, indicating that they were either subtle or based on chance findings.

The potentially OT-predictive value of longer time to ISW requires further investigation in children and adults through a systematic review with meta-analysis. It is possible that LT recipients that do not suffer from acute IS co-morbidity can benefit from delaying the initiation of ISW. Mechanistically, prolonged exposure to donor antigens and increased immune exhaustion can potentially explain the positive effects of delayed ISW start on withdrawal success. ${ }^{7}$ Consistently, the A-WISH trial, which did not meet our eligibility criteria due to belowthreshold proportion of NINV LT recipients (Table S2), examined early ISW ( $1-2$ years posttransplant) and found a relatively low incidence of OT $(10 / 77,13 \%)$ and no prognostic value of time between LT and ISW. ${ }^{64}$

Bearing in mind that non-invasiveness increases the practical utility of a biomarker (and decreases the risk of complications), we synthesized the reports on non-invasive biomarkers separately. PBMC signatures that may reflect immune exhaustion such as high TEMRA+ and Eomes+ CD8+ T cells, ${ }^{34} \mathrm{CD} 4+\mathrm{PD}-1+$ cells, ${ }^{45}$ or CD4+ CD57+ CD45RA- CCR7+ cells, ${ }^{46}$ and low naïve CD8+ T cells ${ }^{34}$ may be good candidate biomarkers to predict OT. Similarly, the promising results of an in vitro lymphocyte proliferation assay ${ }^{26}$ warrant independent implementation in both pediatric and adult ISW studies and may be interpreted as a readout of immune exhaustion. 
Analysis of invasive biomarkers revealed differences between children and adults. Accordingly, a transcriptional predisposition pattern for adult $\mathrm{OT}^{25}$ did not show any prognostic value for pediatric OT. ${ }^{35}$ Information on OTpredictive pediatric gene signatures, which likely exist, is still missing completely. Identified OT-predictive histological markers also conspicuously differed between pediatric and adult ISW candidates. Absence of portal inflammation was found to be OT-predictive in children, ${ }^{14,35}$ whereas an adult study found no prognostic value for this parameter. ${ }^{25}$ Furthermore, presence in liver tissue of fewer APC:lymphocyte pairs was OT-predictive in pediatric but non-prognostic in adult ISW candidates, whereas the opposite was true for an increased load of CD4+ cells. ${ }^{34,35}$

To best highlight the main review findings, we rated the overall value of different predictors based on our data and by expert opinion. Association of two invasive biomarkers with OT, namely no portal inflammation in children and allograft [CDHR2, MIF, PEBP1, SOCS1, TFRC] expression in adults, was rated strong, whereas association of several non-invasive adult biomarkers was deemed moderate only (Table 2). Similarly, the association of longer time between LT and ISW with OT was rated moderate (Table 1). Together, we hope that these preliminary overall judgements will help identify the next step towards reliable ISW prediction.

Given the fact that the best-documented and most promising biomarkers were all invasive (Table 2), our review strongly endorses the necessity of pre-ISW biopsies. The majority of included ISW trials did perform pre-ISW biopsies to assess liver histology (Table S3). Still, 8/23 publications did not report any pre-ISW or post-ISW liver histology findings. A liver biopsy is crucial for assessing subtle graft inflammation and fibrosis and patients' eligibility for ISW. According to the latest Banff update, ${ }^{65}$ pre-ISW biopsy findings that focus on signs of subtle graft injury can be associated with ISW failure. In Figure 3, we have drafted a two-step selection algorithm for NINV LT recipients towards ISW that takes into account the inclusion/exclusion criteria of current ISW trials, ${ }^{49,53}$ the Banff recommendations, ${ }^{65}$ and our review findings (Table 2).

Our secondary outcomes - the ISW success rate and the number of graft losses - showed comparable results in children and adults (Tables S6 and S7). In highly selected ISW cohorts, the fraction of successful ISW is usually $>30 \%$. A general advantage of children resulting in presumable higher OT rates ${ }^{8}$ could not be confirmed. At the 
same time, graft loss very rarely occurred, indicating that IS reinstitution in response to ISW failure is mostly successful. We conclude that the risk of ISW in well-structured trials is relatively low, although there is a per se risk in the setting of every clinical trial that includes frequent and invasive monitoring. The current AASLD 66,67 and EASL ${ }^{68}$ guidelines recommend a rigorous selection of candidates (see also Figure 3 ) and close monitoring. EASL and AASLD for children further suggest restricting ISW to trials. ${ }^{66,68}$ Future guideline updates will give more guidance for clinicians to perform ISW. 


\section{Limitations}

We acknowledge the following study limitations. (i) The present review exclusively included ISW studies that assessed and reported pre-ISW factors. While the clean presentation of OT-predictors is a strength, the exclusion of important ISW studies that assessed, e.g., biomarkers after OT achievement renders the mapping of ISW studies incomplete. (ii) Our results are irrelevant for ISW candidates with active viral hepatitis - one of our exclusion criteria - since allograft environment and ISW outcomes in these patients are evidently distinct. ${ }^{25,69,70}$ (iii) Our finding that IS regimen appears not to impact OT outcome is likely limited by data availability. Indeed, ISW candidates on mTOR-I treatments display several tolerogenic features ${ }^{71}$ and a relatively high OT success rate of $>50 \%$ in a single-arm study. ${ }^{34}$ (iv) Our eligibility criteria did not consider different criteria for patient inclusion into ISW studies, which led to some inter-study heterogeneity, e.g. in the times between LT and ISW (Tables S6 and S7) or the duration of follow-up (Table S3). (v) By limiting our study to spontaneous OT, the important research on OT-inducing treatments was not assessed. ${ }^{4}$ (vi) We also included conference abstracts, which provide only limited data detail and are lacking the quality control of peer-review. 


\section{Conclusions}

Our results highlight the need for validation and further development as well as new discovery of predictive biomarkers, since clinical parameters - with potential exception of time to ISW - appear to be of limited value to predict OT in LT recipients. The ideal biomarker would have to meet many criteria including being easy and rapid to assess, accurate, with high sensitivity and specificity, reproducible across centers, and affordable. While it is unlikely that a single biomarker will fulfill all these criteria, the results of the first prospective multicenter biomarker-guided ISW studies in adults ${ }^{49,53}$ (Table S5) are eagerly awaited. Next to these ongoing validation efforts, all biomarkers demonstrating prognostic value in stand-alone studies (Table 2) will require further assessment and cross-cohort validation. Our comprehensive mapping of biomarkers, including those that are relevant in children, will help guide the design of such follow-up studies. Strong candidates for biomarkers to be scrutinized are allograft gene expression signatures, histopathological scores, and immune exhaustion markers. 
Acknowledgements / Funding

We thank Hannah Ewald for peer-review of the search strategies and Sandy Feng for the sharing of unpublished data. JV is supported by the Swiss National Science Foundation (grants P2LAP3_181318 and P400PM_194501). 


\section{Disclosure}

The authors of this manuscript have no conflicts of interest to disclose as described by the American Journal of Transplantation. 
Data availability statement

Data sharing is not applicable to this article as no new data were created or analyzed in this study. 


\section{Figure legends}

Figure 1: Timeline of immunosuppression withdrawal in liver transplant recipients. Following LT (elapsed time variable), allograft recipients can be selected for ISW. Selection is guided by the likelihood of later ISW success (or OT), which can be assessed by liver function tests, biopsy findings, and biomarker assessment. LT recipients with unfavorable prognostic markers at baseline are deemed non-eligible. ISW takes 6-12 months until complete withdrawal (End of ISW) and OT is defined as rejection-free liver allograft survival for at least one year following ISW. Typically during these periods, a majority of patients experience allograft rejection and require reinstitution of IS (ISW failure). ISW success is documented with normal liver function tests and a liver biopsy that confirms the absence of rejection, and requires long-term follow-up.

Figure 2: Study flow diagram for the selection of articles, conference abstracts, and trial registry entries.

Figure 3: Proposition for a two-step selection algorithm towards immunosuppression withdrawal in nonautoimmune/non-replicative viral liver transplant recipients. Eligibility of LT recipients for ISW should be guided by patient history and liver biopsy. ISW candidates that fulfil the screening criteria (left text box) are subjected to a liver biopsy and detailed histologic analysis (right text box). For adults, also gene expression profile analysis of biopsies should be considered. Candidates that show favorable allograft histology can start ISW. The proposed algorithm is based on inclusion/exclusion criteria of current ISW trials, ${ }^{49,53}$ the latest Banff update, ${ }^{65}$ and the findings in this review (in italic letters). It should be noted that many details of OT-predictive allograft gene expression profiles (e.g. Which exact gene combinations? Which experimental platforms to quantify gene expression?) are still under active research. ${ }^{52,53} \mathrm{AlH}$, autoimmune hepatitis; PBC, primary biliary cirrhosis; PSC, primary sclerosing cholangitis. 
Supporting information statement

Additional supporting information may be found online in the Supporting Information section at the end of the article. 


\section{References}

1. Chak E, Saab S. Risk factors and incidence of de novo malignancy in liver transplant recipients: a systematic review. Liver International 2010;30(9):1247-1258.

2. Ojo AO, Held PJ, Port FK, Wolfe RA, Leichtman AB, Young EW et al. Chronic renal failure after transplantation of a nonrenal organ. The New England journal of medicine 2003;349(10):931-940.

3. Memaran N, Borchert-Mörlins B, Schmidt BMW, Sugianto RI, Wilke H, Blöte R et al. High Burden of Subclinical Cardiovascular Target Organ Damage After Pediatric Liver Transplantation. Liver Transplantation 2019;25(5):752-762.

4. Mathew JM, Leventhal JR. Clinical transplant tolerance: Coming of age. Human Immunology 2018;79(5):255-257.

5. Heymann F, Tacke F. Immunology in the liver--from homeostasis to disease. Nature reviews Gastroenterology \& hepatology 2016;13(2):88-110.

6. Demetris AJ, Bellamy CO, Gandhi CR, Prost S, Nakanuma Y, Stolz DB. Functional Immune Anatomy of the Liver-As an Allograft. American Journal of Transplantation 2016;16(6):1653-1680.

7. Thomson AW, Vionnet J, Sanchez-Fueyo A. Understanding, predicting and achieving liver transplant tolerance: from bench to bedside. Nature reviews Gastroenterology \& hepatology 2020.

8. Levitsky J, Feng S. Tolerance in clinical liver transplantation. Human Immunology 2018;79(5):283-287.

9. Vionnet J, Sánchez-Fueyo A. Biomarkers of immune tolerance in liver transplantation. Human Immunology 2018;79(5):388-394.

10. Peters MDJ, Godfrey C, McInerney P, Munn Z, Tricco AC, Khalil H. Chapter 11: Scoping Reviews (2020 version). JBI Manual for Evidence Synthesis 2020; Available from:

https://wiki.joannabriggs.org/display/MANUAL/Chapter+11\%3A+Scoping+reviews

11. Tricco AC, Lillie E, Zarin W, O'Brien KK, Colquhoun H, Levac D et al. PRISMA Extension for Scoping Reviews (PRISMA-ScR): Checklist and Explanation. Annals of internal medicine 2018;169(7):467-473.

12. Appenzeller-Herzog $\mathrm{C}$, Hartleif $\mathrm{S}$, Vionnet J. Clinical parameters and biomarkers predicting spontaneous operational tolerance after liver transplantation: a scoping review protocol. F1000Res 2019;8:2059.

13. Baroja-Mazo A, Revilla-Nuin B, de Bejar Á, Martínez-Alarcón L, Herrero Jl, El-Tayeb A et al. Extracellular adenosine reversibly inhibits the activation of human regulatory $T$ cells and negatively influences the achievement of the operational tolerance in liver transplantation. American Journal of Transplantation 2019;19(1):48-61.

14. Feng S, Ekong UD, Lobritto SJ, Demetris AJ, Roberts JP, Rosenthal P et al. Complete immunosuppression withdrawal and subsequent allograft function among pediatric recipients of parental living donor liver transplants. JAMA - Journal of the American Medical Association 2012;307(3):283-293.

15. Millán O, Benitez C, Guillén D, López A, Rimola A, Sánchez-Fueyo A et al. Biomarkers of immunoregulatory status in stable liver transplant recipients undergoing weaning of immunosuppressive therapy. Clinical Immunology 2010;137(3):337-346.

16. Pons JA, Ramírez P, Revilla-Nuin B, Pascual D, Baroja-Mazo A, Robles R et al. Immunosuppression withdrawal improves long-term metabolic parameters, cardiovascular risk factors and renal function in liver transplant patients. Clinical Transplantation 2009;23(3):329-336.

17. Takatsuki $\mathrm{M}$, Uemoto $\mathrm{S}$, Inomata $\mathrm{Y}$, Egawa $\mathrm{H}$, Kiuchi T, Fujita $\mathrm{S}$ et al. Weaning of immunosuppression in living donor liver transplant recipients. Transplantation 2001;72(3):449-454.

18. Nafady-Hego H, Li Y, Ohe H, Elgendy H, Zhao X, Sakaguchi S et al. Utility of CD127 combined with FOXP3 for identification of operational tolerance after liver transplantation. Transplant Immunology 2016;36:1-8.

19. Benítez C, Londoño MC, Miquel R, Manzia TM, Abraldes JG, Lozano JJ et al. Prospective multicenter clinical trial of immunosuppressive drug withdrawal in stable adult liver transplant recipients. Hepatology 2013;58(5):1824-1835.

20. Ohe H, Waki K, Yoshitomi M, Morimoto T, Nafady-Hego H, Satoda N et al. Factors affecting operational tolerance after pediatric living-donor liver transplantation: Impact of early post-transplant events and HLA match. Transplant International 2012;25(1):97-106. 
21. Pons JA, Yélamos J, Ramírez $P$, Oliver-Bonet $M$, Sánchez $A$, Rodríguez-Gago $M$ et al. Endothelial cell chimerism does not influence allograft tolerance in liver transplant patients after withdrawal of immunosuppression. Transplantation 2003;75(7):1045-1047.

22. Waki K, Sugawara Y, Mizuta K, Taniguchi M, Ozawa M, Hirata M et al. Predicting operational tolerance in pediatric living-donor liver transplantation by absence of HLA antibodies. Transplantation 2013;95(1):177-

183.

23. Lin NC, Wang HK, Yeh YC, Liu CP, Loong CC, Tsai HL et al. Minimization or withdrawal of immunosuppressants in pediatric liver transplant recipients. Journal of Pediatric Surgery 2015;50(12):21282133.

24. Pons JA, Revilla-Nuin B, Baroja-Mazo A, Ramírez P, Martínez-Alarcón L, Sánchez-Bueno F et al. FoxP3 in peripheral blood is associated with operational tolerance in liver transplant patients during immunosuppression withdrawal. Transplantation 2008;86(10):1370-1378.

25. Bohne F, Martínez-Llordella M, Lozano JJ, Miquel R, Benítez C, Londoño MC et al. Intra-graft expression of genes involved in iron homeostasis predicts the development of operational tolerance in human liver transplantation. Journal of Clinical Investigation 2012;122(1):368-382.

26. De La Garza RG, Sarobe P, Merino J, Lasarte JJ, D'Avola D, Belsue V et al. Trial of complete weaning from immunosuppression for liver transplant recipients: Factors predictive of tolerance. Liver Transplantation 2013;19(9):937-944.

27. Nafady-Hego H, Li Y, Ohe H, Zhao X, Satoda N, Sakaguchi S et al. The generation of donor-specific CD4+CD25 ++CD45RA+ naive regulatory $T$ cells in operationally tolerant patients after pediatric living-donor liver transplantation. Transplantation 2010;90(12):1547-1555.

28. García de la Garza R, Sarobe P, Merino J, Lasarte JJ, D'Avola D, Belsue V et al. Immune monitoring of immunosuppression withdrawal of liver transplant recipients. Transplant Immunology 2015;33(2):110-116.

29. Perez-Sanz F, Revilla-Nuin B, Marinez-Alarcon L, Herrero JI, Ramirez P, Pons JA et al. Tolerance Biomarkers in Liver Transplantation: Independent External Validation of the Predictive Strength of SENP6 and FEM1C Gene Expression. Transplantation 2019;30:30.

30. Bonaccorsi-Riani E, Pennycuick A, Londono MC, Lozano JJ, Benitez C, Sawitzki B et al. Molecular Characterization of Acute Cellular Rejection Occurring During Intentional Immunosuppression Withdrawal in Liver Transplantation. American Journal of Transplantation 2016;16(2):484-496.

31. Talisetti A, Hurwitz M, Sarwal M, Berquist W, Castillo R, Bass D et al. Analysis of clinical variables associated with tolerance in pediatric liver transplant recipients. Pediatric Transplantation 2010;14(8):976-979.

32. Revilla-Nuin B, de Bejar Á, Martínez-Alarcón L, Herrero JI, Martínez-Cáceres CM, Ramírez P et al. Differential profile of activated regulatory $T$ cell subsets and microRNAs in tolerant liver transplant recipients. Liver Transplantation 2017;23(7):933-945.

33. Waki K, Sugawara Y, Mizuta K, Fujita H, Kadowaki T, Kokudo N. Living-donor liver transplantation at the University of Tokyo, 1996-2011: the impact of HLA matching and a positive crossmatch on long-term survival and tolerance. Clinical transplants 2011:223-235.

34. Levitsky J, Burrell BE, Kanaparthi S, Turka LA, Kurian S, Sanchez-Fueyo A et al. Immunosuppression Withdrawal in Liver Transplant Recipients on Sirolimus. Hepatology 2019.

35. Feng S, Bucuvalas JC, Mazariegos GV, Magee JC, Sanchez-Fueyo A, Spain KM et al. Efficacy and Safety of Immunosuppression Withdrawal in Pediatric Liver Transplant Recipients: Moving Towards Personalized Management. Hepatology 2020.

36. Gu G, Lu T, Xu N, Xia Q. Liver transplant tolerance induced by severe or consistent infection revealed the association with T cell exhaustion. Transplantation 2018;102(5):112.

37. Sanchez-Fueyo A, Douiri A, Elstad M, Kodela E, Wall J, Riani EB et al. Prevalence of subclinical histological lesions and tolerance biomarkers in long-term adult liver transplant recipients considered for immunosuppression withdrawal. Journal of Hepatology 2018;68:S27.

38. Chandran S, Abecassis M, Levitsky J, Feng S, Humar A, Emond J et al. Subclinical histologic findings are observed in $25 \%$ of stable adult liver transplant recipients (ALTRs) screened for immunosuppression withdrawal (ISW): OPTIMAL (NCT02533180). American Journal of Transplantation 2017;17:778-779.

39. Chruscinski A, Rojas-Luengas V, Macarthur M, Issachar A, Luo J, Yowanto H et al. Results of litmus (NCT 02541916): The liver immune tolerance biomarker utilization study. Transplant International 2017;30:108. 
40. Chruscinski A, Rojas-Luengas V, Issacher A, Luo J, Yowanto H, Selzner $\mathrm{N}$ et al. Results of litmus (nct 02541916): The liver immune tolerance bio marker utilization study. American Journal of Transplantation 2018;18:477-478.

41. Vionnet J, Mxquel R, Douiri A, Elstad M, Kodela E, QuagHa A et al. Identification of adult liver transplant recipients eligible to participate in an immunosuppression withdrawal trial employing non-invasive assessments of allograft status. American Journal of Transplantation 2019;19:1165.

42. Ramaswami B, Osamu Y, Ippolito R, Metes D, Zeevi A, Thomson A et al. Operationally tolerant pediatric liver transplant recipients exhibit features of immune activation and exhaustion. Transplantation 2014;98:231. 43. Danger R, Lozano J, Bonaccorsi-Riani E, Kodela E, Mas-Malavila R, Londono MC et al. Hepatocyterelated miR-193a-3p is over-expressed in allograft biopsy from liver tolerant recipients. American Journal of Transplantation 2015;15.

44. Ramaswami B, Yoshida O, Ippolito R, Metes D, Zeevi A, Thomson A et al. Pediatric liver transplant recipients with operational tolerance exhibit features of immune activation and exhaustion. Journal of Immunology 2014;192(1).

45. Burrell B, Feng S, Bridges N, Ekong U, Kanaparthi S, Kirk A et al. Tolerant pediatric liver transplant (LT) recipients (TOL) from WISP-R (NCT00320606) split into two phenotypically distinct groups by PD1 expression on CD4+ T cells at baseline, prior to immunosuppression withdrawal (ISW). American Journal of Transplantation 2017;17:275-276.

46. Ramaswami B, Yoshida O, Ippolito R, Marrari M, Bentlejewski C, Metes D et al. Pediatric liver transplant recipients with operational tolerance exhibit features of immune activation and exhaustion. Transplantation 2015;99(7):115-116.

47. Feng S, Tchao N, Ekong U, Stempora L, Kirk A, Turka L et al. High percentage of PD-1+CD4+ T cells in a subset of tolerant pediatric liver recipients. Transplantation 2014;98:872.

48. Chruscinski A, Rojas-Luengas V, Macarthur M, Issacher A, Luo J, Yowanto $\mathrm{H}$ et al. Results of LITMUS (NCT 02541916): The liver immune tolerance bio marker utilization study. Transplantation 2018;102(5):307308.

49. NCT02533180. Evaluation of Donor Specific Immune Senescence and Exhaustion as Biomarkers of Tolerance Post Liver Transplantation. 2015; Available from: https://clinicaltrials.gov/show/NCT02533180

50. NCT01445236. Pilot Study of Immunosuppression Drug Weaning in Liver Recipients Exhibiting Biomarkers of High Likelihood of Tolerance. 2011; Available from: http://clinicaltrials.gov/show/NCT01445236 51. NCT02743793. A Cohort Study of Operationally Tolerant Allograft Recipients. 2016; Available from: https://ClinicalTrials.gov/show/NCT02743793

52. NCT02541916. Liver Immune Tolerance Marker Utilization Study. 2015; Available from: https://ClinicalTrials.gov/show/NCT02541916

53. NCT02498977. Liver Immunosuppression Free Trial. 2015; Available from: https://ClinicalTrials.gov/show/NCT02498977

54. Castellaneta A, Mazariegos GV, Nayyar N, Zeevi A, Thomson AW. HLA-G level on monocytoid dendritic cells correlates with regulatory T-cell Foxp3 expression in liver transplant tolerance. Transplantation 2011;91(10):1132-1140.

55. Tokita D, Mazariegos GV, Zahorchak AF, Chien N, Abe M, Raimondi G et al. High PD-L1/CD86 ratio on plasmacytoid dendritic cells correlates with elevated T-regulatory cells in liver transplant tolerance.

Transplantation 2008;85(3):369-377.

56. Li Y, Zhao X, Cheng D, Haga H, Tsuruyama T, Wood $\mathrm{K}$ et al. The presence of Foxp3 expressing T cells within grafts of tolerant human liver transplant recipients. Transplantation 2008;86(12):1837-1843.

57. Höfer A, Jonigk D, Hartleben B, Verboom M, Hallensleben M, Manns MP et al. Non-invasive screening for subclinical liver graft injury in adults via donor-specific anti-HLA antibodies. In. Scientific reports. 2020: 14242.

58. Xie L, Ichimaru N, Morita M, Chen J, Zhu P, Wang J et al. Identification of a novel biomarker gene set with sensitivity and specificity for distinguishing between allograft rejection and tolerance. Liver Transplantation 2012;18(4):444-454. 
59. Feng S, Demetris AJ, Spain KM, Kanaparthi S, Burrell BE, Ekong UD et al. Five-year histological and serological follow-up of operationally tolerant pediatric liver transplant recipients enrolled in WISP-R. Hepatology 2017;65(2):647-660.

60. Maria Manzia T, Angelico R, Toti L, Angelico C, Quaranta C, Parente A et al. Longterm Survival and CostEffectiveness of Immunosuppression Withdrawal After Liver Transplantation. Liver Transplantation 2018;24(9):1199-1208.

61. Mazariegos GV, Zahorchak AF, Reyes J, Chapman H, Zeevi A, Thomson AW. Dendritic cell subset ratio in tolerant, weaning and non-tolerant liver recipients is not affected by extent of immunosuppression. American Journal of Transplantation 2005;5(2):314-322.

62. Zarkhin V, Talisetti A, Li L, Wozniak L, McDiarmid SV, Cox K et al. Expression of soluble HLA-G identifies favorable outcomes in liver transplant recipients. Transplantation 2010;90(9):1000-1005.

63. Zhao X, Li Y, Ohe H, Nafady-Hego H, Uemoto S, Bishop GA et al. Intragraft $V \delta 1 \gamma \delta$ T cells with a unique $\mathrm{T}$-Cell receptor are closely associated with pediatric semiallogeneic liver transplant tolerance. Transplantation 2013;95(1):192-202.

64. Shaked A, DesMarais MR, Kopetskie H, Feng S, Punch JD, Levitsky J et al. Outcomes of immunosuppression minimization and withdrawal early after liver transplantation. American Journal of Transplantation 2019;19(5):1397-1409.

65. Demetris AJ, Bellamy C, Hubscher SG, O'Leary J, Randhawa PS, Feng S et al. 2016 Comprehensive Update of the Banff Working Group on Liver Allograft Pathology: Introduction of Antibody-Mediated Rejection. American Journal of Transplantation 2016;16(10):2816-2835.

66. Kelly DA, Bucuvalas JC, Alonso EM, Karpen SJ, Allen U, Green M et al. Long-term medical management of the pediatric patient after liver transplantation: 2013 practice guideline by the American Association for the Study of Liver Diseases and the American Society of Transplantation. Liver Transplantation 2013;19(8):798825.

67. Lucey MR, Terrault N, Ojo L, Hay JE, Neuberger J, Blumberg E et al. Long-term management of the successful adult liver transplant: 2012 practice guideline by the American Association for the Study of Liver Diseases and the American Society of Transplantation. Liver Transplantation 2013;19(1):3-26.

68. European Association for the Study of the Liver. Electronic address eee. EASL Clinical Practice Guidelines: Liver transplantation. Journal of Hepatology 2016;64(2):433-485.

69. Bohne F, Londoño MC, Benítez C, Miquel R, Martínez-Llordella M, Russo C et al. HCV-induced immune responses influence the development of operational tolerance after liver transplantation in humans. Science Translational Medicine 2014;6(242).

70. Orlando G, Manzia T, Baiocchi L, Sanchez-Fueyo A, Angelico M, Tisone G. The Tor Vergata weaning off immunosuppression protocol in stable HCV liver transplant patients: The updated follow up at 78 months. Transplant Immunology 2008;20(1-2):43-47.

71. Levitsky J, Mathew JM, Abecassis M, Tambur A, Leventhal J, Chandrasekaran D et al. Systemic immunoregulatory and proteogenomic effects of tacrolimus to sirolimus conversion in liver transplant recipients. Hepatology 2013;57(1):239-248. 


\section{Table 1: Association of pre-immunosuppression withdrawal clinical parameters with OT}

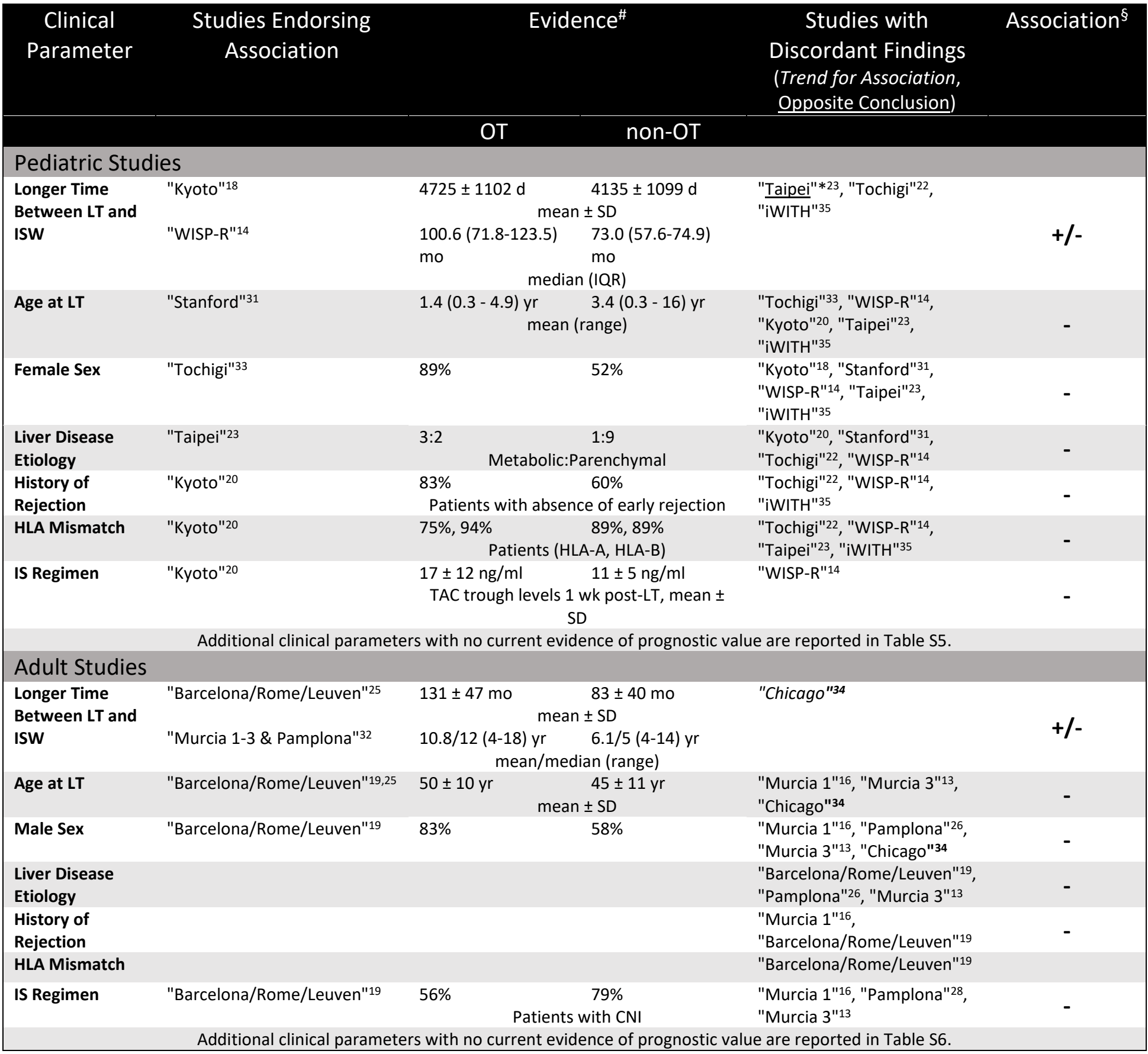

\# From report with largest sample size, * For numerical evidence, see Table S4, § Expert opinion: +, strong; +/-, moderate; -, weak

CNI, calcineurin inhibitor; d, days; HLA, human leukocyte antigen; IQR, interquartile range; IS, immunosuppression; ISW, immunosuppression withdrawal; LT, liver transplantation; ml, milliliters; mo, months; ng, nanograms; SD, standard deviation; wk, weeks; yr, years 


\section{Table 2: Association of pre-immunosuppression withdrawal biomarkers with OT}

\begin{tabular}{|l|ccc|c|}
\hline Biomarker & $\begin{array}{c}\text { Studies Endorsing } \\
\text { Association }\end{array}$ & Evidence & $\begin{array}{c}\text { Studies with } \\
\text { Discordant Findings } \\
\text { (Trend for Association) }\end{array}$ & Association \\
& OT & non-OT & &
\end{tabular}

\section{Pediatric Studies}

Non-Invasive Biomarkers

Lower Anti-HLA Antibodies

(incl. DSA)

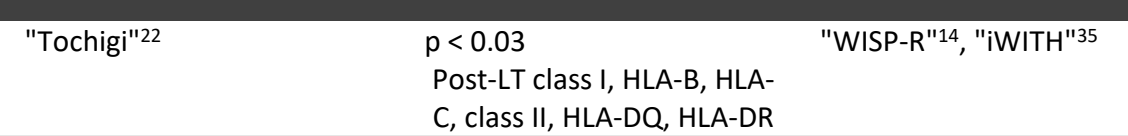

Additional non-invasive biomarkers with no current evidence of prognostic value are reported in Table S5.

Invasive Biomarkers

No Portal Inflammation

"WISP-R"14

$91.7(61.5-$

$42.9(9.9-$

99.8)\% 81.6)\%

Patients $(95 \mathrm{Cl})$

"iWITH"35 $0.36(0.14-0.90)$

Mild vs No Portal

Inflammation, OR (95Cl)

\begin{tabular}{|c|c|c|c|}
\hline $\begin{array}{l}\text { Fewer APC:Lymphocyte } \\
\text { Pairs }\end{array}$ & "iWITH"35 & \multicolumn{2}{|c|}{$0.82(0.74-0.92)$} \\
\hline Lower C4d Score & "WISP-R"14 & $6.1(5.1-9.3)$ & 12.5 \\
\hline & & \multicolumn{2}{|c|}{ median (IQR } \\
\hline Lower Load of Leukocytes & "iWITH"35 & \multicolumn{2}{|c|}{$0.97(0.95-0.99)$} \\
\hline $\begin{array}{l}\text { Lower Load of Infiltrating } \\
\text { Monocytes/Macrophages }\end{array}$ & "iWITH"35 & \multicolumn{2}{|c|}{$0.91(0.85-0.97)$} \\
\hline
\end{tabular}

[CDHR2, MIF, PEBP1,

SOCS1, TFRC] Expression in

Allograft

Adult Studies

Non-Invasive Biomarkers

Lower Lymphocyte

Proliferation

NK Cells Higher in PBMC

\section{V $\delta 2-T C R ~ \gamma \delta$ T Cells Higher in}

PBMC

"Tolerogenic DC" Higher in

PBMC

Naive CD8+ Cells Lower in

PBMC

TEMRA CD8+ Cells Higher

in PBMC

Eomes+ CD8+ Cells Higher

in PBMC

Higher FEM1C Expression in

PBMC

Higher SENP6 Expression in

PBMC

Higher miR95 Expression in

PBMC

Higher miR31 Expression in

PBMC
"Pamplona"26,28

$7.5(2.1-23) \quad 41.7(19-65)$

In vitro Stimulated

Lymphocyte Proliferation

Index, median (IQR)
"Barcelona/Rome/Leuven"25 NR

"Pamplona"26,28

$14.0(9.9-\quad 8.3(6.0-$

20.7)\% 13.7)\%

median (IQR), $\mathrm{p}=0.07$

$p=0.03$

$p<0.01$

$p<0.01$

$p<0.05$

$p<0.05$

"Chicago" 34

"Murcia 3"29

AUC 0.967

AUC 0.933

AUC 0.867

AUC 0.967

"Murcia 3"29

"Murcia 3"29
$+/-$

"iWITH"35
$+/-$

$+/-$

"Murcia 3"29
"Murcia 1"24, "Chicago"34

$$
+/-
$$

"Murcia 1"24, "Murcia 3"29

+ -

+ /-

$+/-$

$+/-$

$+/-$

+ /-

$+/-$

$+/-$

$+/-$ 


\begin{tabular}{|c|c|c|c|c|}
\hline Higher Serum Ferritin & "Barcelona/Rome/Leuven"25 & $\begin{array}{ll}185.5(26- & 73.5(3-304) \\
864) \mathrm{ng} / \mathrm{ml} & \mathrm{ng} / \mathrm{ml} \\
\quad \text { mean (range) }, \mathrm{p}<0.01\end{array}$ & "Murcia 3"29 & $+/-$ \\
\hline Lower Cytokine Secretion & "Barcelona/Rome/Leuven"15 & $\begin{aligned} & \mathrm{p}<0.05 \\
& \quad \text { In vitro IFN } \gamma \text { secretion }\end{aligned}$ & "Pamplona"28 & - \\
\hline Treg Cells Higher in PBMC & & & $\begin{array}{l}\text { "Murcia 1"24, } \\
\text { "Barcelona/Rome/Leuven"25, } \\
\text { "Pamplona"26,28, "Murcia } \\
\text { 3"29, "Chicago"34 }\end{array}$ & - \\
\hline $\begin{array}{l}\text { Higher FoxP3 Expression in } \\
\text { PBMC }\end{array}$ & "Barcelona/Rome/Leuven"30 & NR & "Murcia 1"24 & - \\
\hline $\begin{array}{l}\text { [NCR1, PDGFRB, PSMD14] } \\
\text { Expression in PBMC }\end{array}$ & "Barcelona/Rome/Leuven"25 & AUC 0.76 & & - \\
\hline $\begin{array}{l}\text { [SLAMF7, KLRF1, CLIC3, } \\
\text { PSMD14, ALG8, CX3CR1] } \\
\text { Expression in PBMC }\end{array}$ & "Barcelona/Rome/Leuven"25 & AUC 0.71 & & - \\
\hline $\begin{array}{l}\text { Lower Anti-HLA Antibodies } \\
\text { (incl. DSA) }\end{array}$ & & & $\begin{array}{l}\text { "Barcelona/Rome/Leuven"19, } \\
\text { "Chicago"34 }\end{array}$ & - \\
\hline Higher Serum Hepcidin-25 & "Barcelona/Rome/Leuven"25 & $p<0.05$ & "Murcia 3"29 & - \\
\hline \multicolumn{5}{|c|}{ Additional non-invasive biomarkers with no current evidence of prognostic value are reported in Table S6. } \\
\hline \multirow[t]{2}{*}{$\begin{array}{l}\text { [CDHR2, MIF, PEBP1, } \\
\text { SOCS1, TFRC] Expression in } \\
\text { Allograft }\end{array}$} & "Barcelona/Rome/Leuven"25 & $\begin{array}{l}49(7-343) \\
{[89+80 \% \text { SN, } 86+100 \% \text { SP, }} \\
80+100 \% \text { PPV, 92+85\% } \\
\text { NPV, 13+9.5\% ER, AUC } \\
0.83]\end{array}$ & & + \\
\hline & "Chicago"34 & $\begin{array}{l}\quad \text { OR }(95 \mathrm{Cl}) \\
88 \% \text { SN, } 83 \% \text { SP, } 88 \% \text { PPV, } \\
83 \% \text { NPV }\end{array}$ & & \\
\hline Higher Load of CD4+ Cells & "Chicago"34 & $\begin{array}{l}178 \text { (168- } 85 \text { (69-158) } \\
205) \quad \text { median (IQR) }\end{array}$ & & $+/-$ \\
\hline Higher Hepatocytic Iron & "Barcelona/Rome/Leuven"25 & $p<0.01$ & & $+/-$ \\
\hline No Portal Inflammation & & & "Barcelona/Rome/Leuven"25 & - \\
\hline
\end{tabular}

\footnotetext{
$\S$ Expert opinion: +, strong; +/-, moderate; -, weak
}

APC, antigen-presenting cell; AUC, area under the curve; DC, dendritic cell; DSA, donor-specific antibodies; ER, overall error rate; HLA, human leukocyte antigen; IFN $\gamma$, interferon gamma; IQR, interquartile range; ISW, immunosuppression withdrawal; LT, liver transplantation; $\mathrm{ml}$, milliliters; ng, nanograms; NK, natural killer; NPV, negative predictive value; NR, not reported; OR, odds ratio; PBMC, peripheral blood mononuclear cells; PPV, positive predictive value; SN, sensitivity; SP, specificity; Treg, regulatory T-cell; $95 \mathrm{Cl}$, $95 \%$ confidence interval 


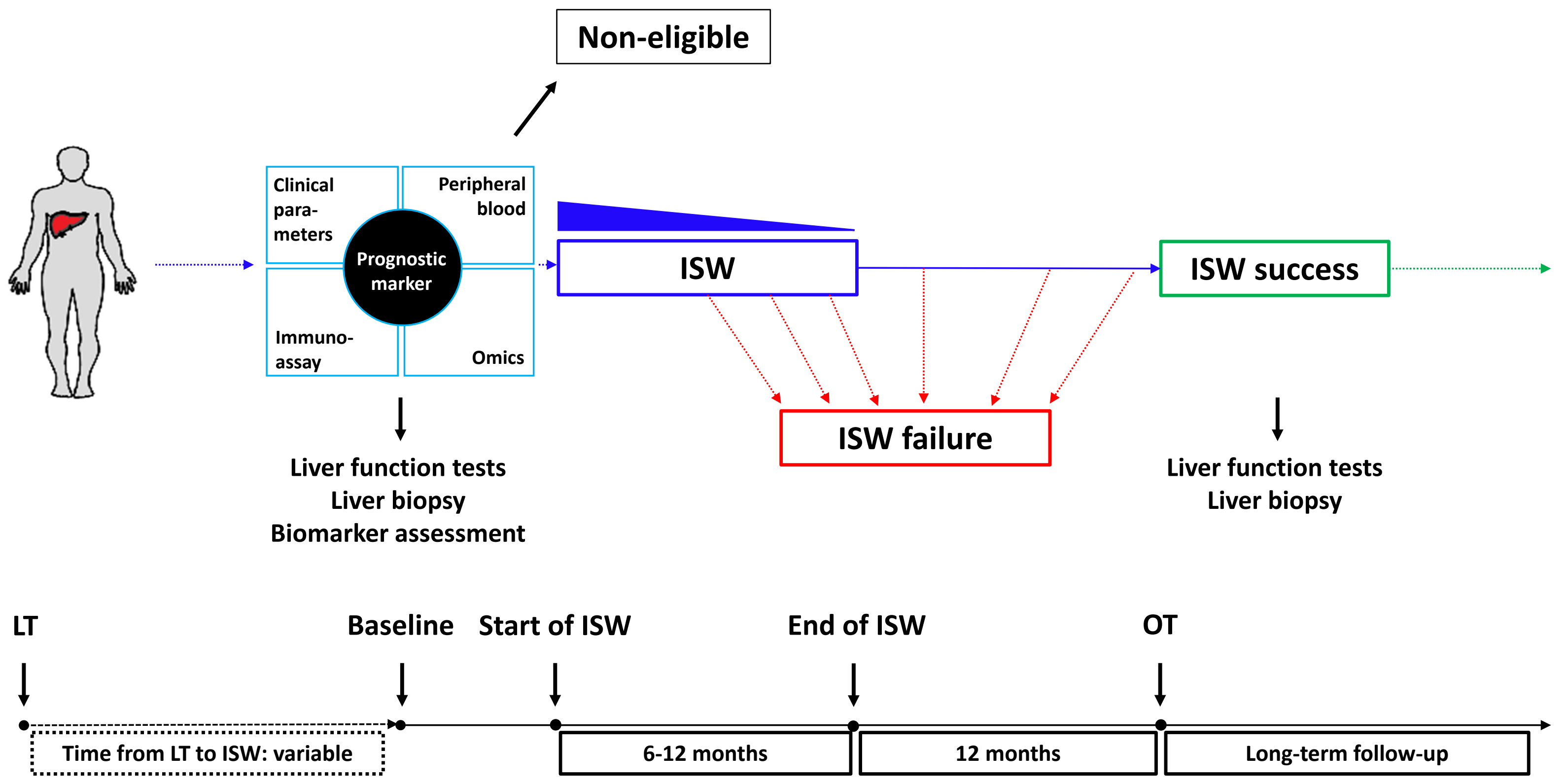




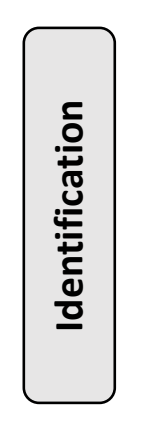

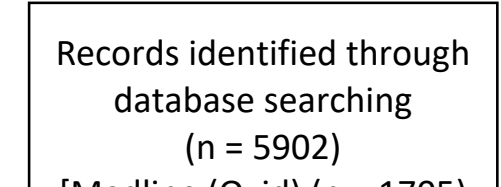

[Medline (Ovid) $(n=1705)$

Embase.com ( $n=3267)$

CENTRAL $(n=930)$ ]
Records identified through

registry searching

$$
(n=104)
$$

[clinicaltrials.gov $(n=82)$

WHO ICTRP $(n=22)]$
Additional records identified through other sources

$$
(n=25)
$$

[from registry entries $(n=2)$

from conference abstracts $(n=6)$

from citations ( $n=16)$

from author contacts $(n=1)$ ]

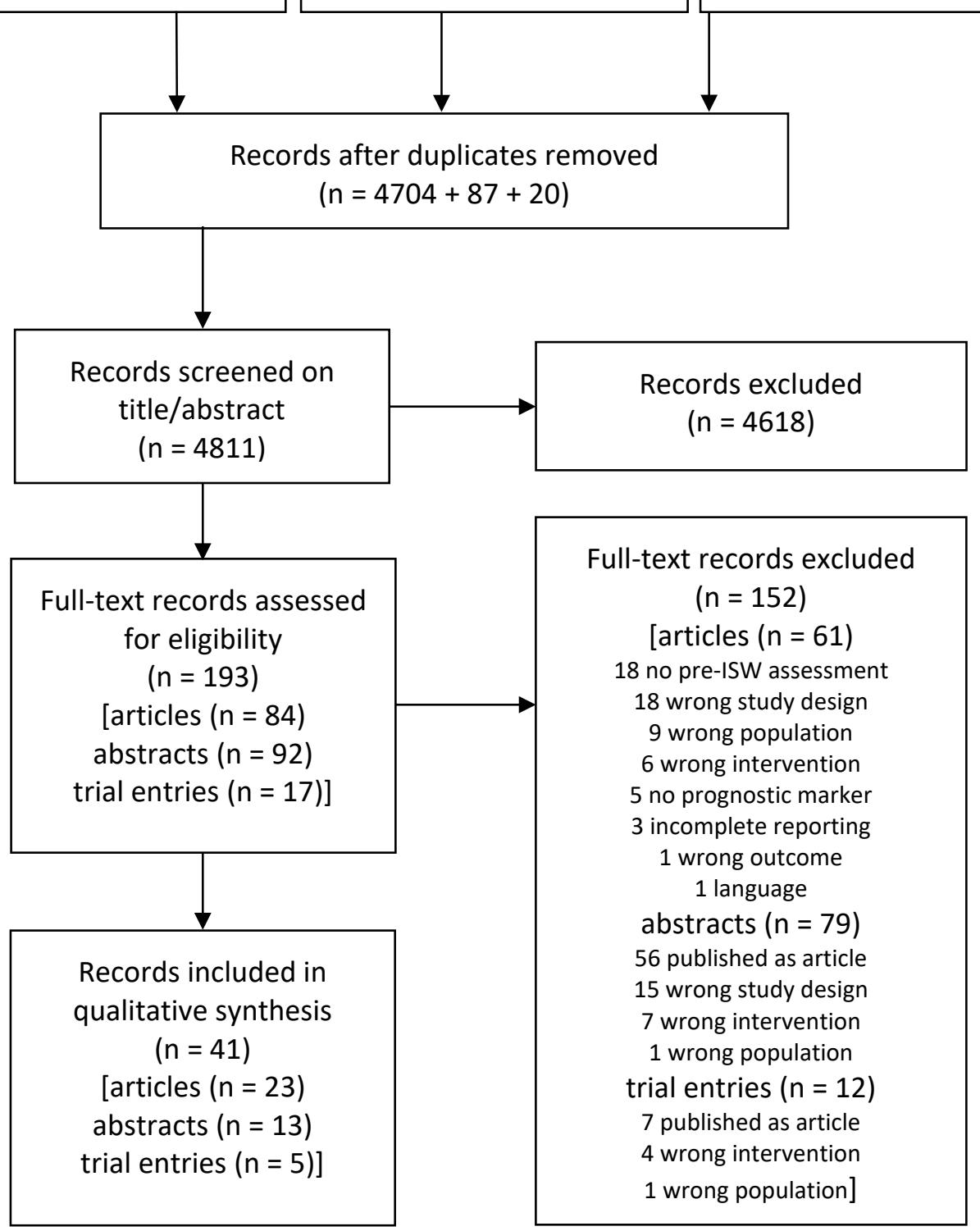

Figure 2 


\section{Screening}

\section{- Inclusion:}

- Normal liver function tests

o > 3-6 years post-transplant

- Monotherapy IS (preferably)

- Compliance to follow-up

- Exclusion if any of the following:

- No recent episodes of acute or chronic rejection ( $<52$ weeks)

- Significant co-morbidities, other than renal injury

- Auto-immune liver disease (AIH, PBC, PSC)

\section{Pre-ISW Biopsy}

- Exclusion if any of the following:

- Portal inflammation (in particular in children) and interface activity

- Centrizonal/perivenular inflammation

- Bile duct changes (unless there is an alternative, non-immunologic explanation)

- Fibrosis (more than mild)

- Arteritis or foam-cell arteriopathy

- Unfavorable allograft gene expression profile (adults)

\section{Non-eligible}




\section{Embase.com}

\section{(20190717; 3,267 hits)}

('liver transplantation'/exp OR (OLT OR LTx):ab,ti OR (('liver'/de OR 'liver lobe'/exp OR 'liver disease'/exp OR 'obstructive bile duct disease'/exp OR 'bile duct atresia'/de OR (liver OR hepatic OR hepato* OR hepatis OR hepatitis OR intrahepatic OR extrahepatic OR cirrhosis OR cirrhotic OR 'periportal fibrosis' OR jaundice OR icterus OR bilirubinaemia OR cholestasis OR cholestatic OR ((bile OR biliary OR choledoch*) NEAR/3 (obstruction OR stasis OR occlusion OR stenosis OR stricture OR obliteration OR atresia OR agenesi*))):ab,ti) AND ('transplantation'/de OR 'organ transplantation'/de OR 'allotransplantation'/de OR 'orthotopic transplantation'/de OR 'recipient'/exp OR (transplant* OR Tx OR allotransplant* OR graft* OR allograft* OR recipient*):ab,ti)))

\section{AND}

((('immunosuppressive agent'/exp OR 'calcineurin inhibitor'/exp OR 'mammalian target of rapamycin inhibitor'/de OR 'immunosuppressive treatment'/de) AND ('treatment withdrawal'/exp OR 'weaning'/de)) OR ((((immunosuppress* OR immuno-suppress* OR immune-suppress* OR immunodepress* OR immuno-depress* OR immune-depress* OR anti-rejection OR antirejection OR 'immune system-suppressing' OR 'transplantation reaction inhibition' OR anti-metaboli* OR antimetaboli* OR azathioprine OR belatacept OR cyclophosphamide OR daclizumab OR 'mycophenolate mofetil' OR MMF OR 'mycophenolic acid' OR cellcept OR 'calcineurin inhibitor*' OR 'protein phosphatase 2B inhibitor*' OR cyclosporin* OR ciclosporin* OR neoral OR sandim* OR tacrolimus OR advagraf OR prograf* OR fk506 OR fk-506 OR 'mammalian target of rapamycin inhibitor*' OR 'mammalian target of rapamycin kinase inhibitor*' OR 'mechanistic target of rapamycin inhibitor*' OR 'mechanistic target of rapamycin kinase inhibitor*' OR 'mTOR inhibitor*' OR 'mTOR kinase inhibitor*' OR everolimus OR rad001* OR rad-001* OR rapamune OR rapamycin OR sirolimus) NEAR/4 (withdraw* OR taper* OR wean* OR minimization OR minimisation OR minimizing OR minimising OR sparing OR eliminat* OR reduction OR reducing OR lower* OR cessation OR discontinu* OR interrupt* OR abstinence OR avoid* OR stop* OR downgrad* OR diminish* OR free*)) $O R$ is-withdraw* $O R$ is-taper* $O R$ is-wean* $O R$ is-minimization $O R$ is-minimisation $O R$ isminimizing $O R$ is-minimising $O R$ is-sparing $O R$ is-eliminat* $O R$ is-reduction $O R$ is-reducing $O R$ islower* $O R$ is-cessation $O R$ is-discontinu* OR is-interrupt* $O R$ is-abstinence $O R$ is-avoid* OR is-stop* $O R$ is-downgrad* $O R$ is-diminish* $O R$ is-free):ab,ti))

\section{AND}

('transplantation tolerance'/de OR 'immunological tolerance'/de OR 'immunoregulation'/de OR 'immunoreactivity'/de OR 'graft survival'/de OR 'liver biopsy'/de OR (tolerogen* OR 'tolerant patient*' OR 'tolerant state' OR 'state of tolerance' OR 'sustained weaning' OR ((transplant* OR posttransplant* OR operational* OR immune OR immunologic* OR alloimmune OR allograft* OR graft* OR alloantigen* OR antigen* OR chimerism OR donor-specific OR peripheral) NEAR/3 (tolerance OR tolerant OR tolerated OR tolerating OR acceptance OR protect* OR quiescen* OR unresponsive* OR nonresponsive* OR un-responsive* OR non-responsive*)) OR immunoregulat* OR immunosurveill* OR immunoreactiv* OR immunoactiv* OR ((immune OR immunologic*) NEXT (regulat* OR surveill* OR reactiv* OR activ*)) OR ((graft OR allograft OR transplant* OR liver OR hepatic) NEAR/3 (survival OR health OR function OR 'resistance to rejection')) OR ((inhibit* OR decrease OR abolish OR suppress* OR reduc* OR ameliorat* OR improve* OR absent OR avoid* OR prevent*) NEAR/3 (graft OR allograft OR transplant* OR liver OR hepatic) NEAR/3 (injury OR complication* OR dysfunction OR inflammation OR fibrosis OR infiltration)) OR ((inhibit* OR decrease OR abolish OR suppress* OR reduc* OR ameliorat* OR improve* OR absent OR avoid* OR prevent*) NEAR/3 (rejection OR 'immune response*' OR 'alloimmune response*' OR 'T-cell response*' OR 'B- 
cell response*' OR 'antibody response*' OR 'humoral response*')) OR ((liver OR hepatic) NEAR/3 (biopsy OR biopsies OR puncture*))):ab,ti)

NOT

(('animal'/de OR 'animal experiment'/exp OR 'nonhuman'/de) NOT ('human'/exp OR 'human experiment'/de))

NOTE: The subject heading "graft rejection" (and respective free text terms) was omitted, because its inclusion resulted in a non-manageable increase of hits.

\section{Medline (Ovid) \\ (20190717; 1,705 hits)}

(liver transplantation/ OR (OLT OR LTx).ab,ti. OR ((liver/ OR exp liver diseases/ OR exp cholestasis/ OR biliary atresia/ OR (liver OR hepatic OR hepato* OR hepatis OR hepatitis OR intrahepatic OR extrahepatic $O R$ cirrhosis $O R$ cirrhotic $O R$ periportal fibrosis $O R$ jaundice $O R$ icterus $O R$ bilirubinaemia OR cholestasis OR cholestatic OR ((bile OR biliary OR choledoch*) ADJ3 (obstruction OR stasis OR occlusion OR stenosis OR stricture OR obliteration OR atresia OR agenesi*))).ab,ti.) AND (transplantation/ OR organ transplantation/ OR transplant recipients/ OR (transplant* OR Tx OR allotransplant* OR graft* OR allograft* OR recipient*).ab,ti.)))

\section{AND}

(((exp immunosuppressive agents/ OR calcineurin inhibitors/ OR exp sirolimus/ OR tacrolimus/ OR cyclosporine/ OR azathioprine/ OR cyclophosphamide/ OR daclizumab/ OR mycophenolic acid/ OR immunosuppression/) AND (ad.fs OR substance withdrawal syndrome/ OR withholding treatment/)) OR (((immunosuppress* OR immuno-suppress* OR immune-suppress* OR immunodepress* OR immuno-depress* OR immune-depress* OR anti-rejection OR antirejection OR immune systemsuppressing $O R$ transplantation reaction inhibition $O R$ anti-metaboli* $O R$ antimetaboli* $O R$ azathioprine OR belatacept OR cyclophosphamide OR daclizumab OR mycophenolate mofetil OR MMF OR mycophenolic acid OR cellcept OR calcineurin inhibitor* OR protein phosphatase 2B inhibitor* OR cyclosporin* OR ciclosporin* OR neoral OR sandim* OR tacrolimus OR advagraf OR prograf* OR fk506 OR fk-506 OR mammalian target of rapamycin inhibitor* OR mammalian target of rapamycin kinase inhibitor* OR mechanistic target of rapamycin inhibitor* OR mechanistic target of rapamycin kinase inhibitor* OR mTOR inhibitor* OR mTOR kinase inhibitor* OR everolimus OR rad001* OR rad-001* OR rapamune OR rapamycin OR sirolimus) ADJ4 (withdraw* OR taper* OR wean* OR minimization OR minimisation OR minimizing OR minimising OR sparing OR eliminat* OR reduction $O R$ reducing $O R$ lower* $O R$ cessation OR discontinu* OR interrupt* $O R$ abstinence $O R$ avoid* OR stop* OR downgrad* OR diminish* OR free*)).ab,ti.))

\section{AND}

(transplantation tolerance/ OR immune tolerance/ OR graft survival/ OR (tolerogen* OR tolerant patient* OR tolerant state OR state of tolerance OR sustained weaning OR ((transplant* OR posttransplant* OR operational* OR immune OR immunologic* OR alloimmune OR allograft* OR graft* OR alloantigen* OR antigen* OR chimerism OR donor-specific OR peripheral) ADJ3 (tolerance OR tolerant OR tolerated OR tolerating OR acceptance OR protect* OR quiescen* OR unresponsive* OR nonresponsive* OR un-responsive* OR non-responsive*)) OR immunoregulat* OR immunosurveill* OR immunoreactiv* OR immunoactiv* OR ((immune OR immunologic*) ADJ 
(regulat* OR surveill* OR reactiv* OR activ*)) OR ((graft OR allograft OR transplant* OR liver OR hepatic) ADJ3 (survival OR health OR function OR resistance to rejection)) OR ((inhibit* OR decrease OR abolish OR suppress* OR reduc* OR ameliorat* OR improve* OR absent OR avoid* OR prevent*) ADJ3 (graft OR allograft OR transplant* OR liver OR hepatic) ADJ3 (injury OR complication* OR dysfunction OR inflammation OR fibrosis OR infiltration)) OR ((inhibit* OR decrease OR abolish OR suppress* OR reduc* OR ameliorat* OR improve* OR absent OR avoid* OR prevent*) ADJ3 (rejection OR immune response* OR alloimmune response* OR T-cell response* OR B-cell response* OR antibody response* OR humoral response*)) OR ((liver OR hepatic) ADJ3 (biopsy OR biopsies OR puncture*))).ab,ti.)

NOT

(exp animals/ NOT humans/)

\section{Cochrane Library (CENTRAL)}

(20190717; Issue 7 of 12, July 2019; 930 hits)

((OLT OR LTX OR ((liver OR hepatic OR hepato* OR hepatis OR hepatitis OR intrahepatic OR extrahepatic OR cirrhosis OR cirrhotic OR 'periportal fibrosis' OR jaundice OR icterus OR bilirubinaemia OR cholestasis OR cholestatic OR ((bile OR biliary OR choledoch*) NEAR/3 (obstruction OR stasis OR occlusion OR stenosis OR stricture OR obliteration OR atresia OR agenesi*))) AND (transplant* OR Tx OR allotransplant* OR graft* OR allograft* OR recipient*))):ab,ti) AND ((((immunosuppress* OR immuno-suppress* OR immune-suppress* OR immunodepress* OR immuno-depress* OR immune-depress* OR anti-rejection OR antirejection OR 'immune systemsuppressing' OR 'transplantation reaction inhibition' OR anti-metaboli* OR antimetaboli* OR azathioprine OR belatacept OR cyclophosphamide OR daclizumab OR 'mycophenolate mofetil' OR MMF OR 'mycophenolic acid' OR cellcept OR 'calcineurin inhibitor*' OR 'protein phosphatase 2B inhibitor*' OR cyclosporin* OR ciclosporin* OR neoral OR sandim* OR tacrolimus OR advagraf OR prograf* OR fk506 OR fk-506 OR 'mammalian target of rapamycin inhibitor*' OR 'mammalian target of rapamycin kinase inhibitor*' OR 'mechanistic target of rapamycin inhibitor*' OR 'mechanistic target of rapamycin kinase inhibitor*' OR 'mTOR inhibitor*' OR 'mTOR kinase inhibitor*' OR everolimus OR rad001* OR rad-001* OR rapamune OR rapamycin OR sirolimus) NEAR/4 (withdraw* OR taper* OR wean* OR minimization OR minimisation OR minimizing OR minimising OR sparing OR eliminat* OR reduction OR reducing OR lower* OR cessation OR discontinu* OR interrupt* OR abstinence OR avoid* OR stop* OR downgrad* OR diminish* OR free*)) OR (is NEXT withdraw*) OR (is NEXT taper*) OR (is NEXT wean*) OR (is NEXT minimization) OR (is NEXT minimisation) OR (is NEXT minimizing) OR (is NEXT minimising) OR (is NEXT sparing) OR (is NEXT eliminat*) OR (is NEXT reduction) OR (is NEXT reducing) OR (is NEXT lower*) OR (is NEXT cessation) OR (is NEXT discontinu*) OR (is NEXT interrupt*) OR (is NEXT abstinence) OR (is NEXT avoid*) OR (is NEXT stop*) OR (is NEXT downgrad*) OR (is NEXT diminish*) OR (is NEXT free)):ab,ti) AND ((tolerogen* OR 'tolerant patient*' OR 'tolerant state' OR 'state of tolerance' OR 'sustained weaning' OR ((transplant* OR posttransplant* OR operational* OR immune OR immunologic* OR alloimmune OR allograft* OR graft* OR alloantigen* OR antigen* OR chimerism OR donor-specific OR peripheral) NEAR/3 (tolerance OR tolerant OR tolerated OR tolerating OR acceptance OR protect* OR quiescen* OR unresponsive* OR nonresponsive* OR un-responsive* OR non-responsive*)) OR immunoregulat* OR immunosurveill* OR immunoreactiv* OR immunoactiv* OR ((immune OR immunologic*) NEXT (regulat* OR surveill* OR reactiv* OR activ*)) OR ((graft OR allograft OR transplant* OR liver OR 
hepatic) NEAR/3 (survival OR health OR function OR 'resistance to rejection')) OR ((inhibit* OR decrease OR abolish OR suppress* OR reduc* OR ameliorat* OR improve* OR absent OR avoid* OR prevent*) NEAR/3 (graft OR allograft OR transplant* OR liver OR hepatic) NEAR/3 (injury OR complication* OR dysfunction OR inflammation OR fibrosis OR infiltration)) OR ((inhibit* OR decrease OR abolish OR suppress* OR reduc* OR ameliorat* OR improve* OR absent OR avoid* OR prevent*) NEAR/3 (rejection OR 'immune response*' OR 'alloimmune response*' OR 'T-cell response*' OR 'Bcell response*' OR 'antibody response*' OR 'humoral response*')) OR ((liver OR hepatic) NEAR/3 (biopsy OR biopsies OR puncture*))):ab,ti)

\section{ClinicalTrials.gov}

(20190903; 82 hits)

tolerance AND liver transplantation (in OTHER TERMS)

\section{WHO ICTRP}

(20190903; 22 hits)

tolerance (in TITLE)

AND

liver transplantation (in CONDITION) 


\begin{tabular}{|c|c|}
\hline No. & Description \\
\hline 1 & Article characteristics \\
\hline $1 a$ & First author \\
\hline $1 b$ & Year of publication \\
\hline 1c & Bibliographic details \\
\hline 2 & Country/ies of study \\
\hline 3 & Trial ID \\
\hline 4 & Study design \\
\hline $4 a$ & Monocenter/multicenter study \\
\hline 4b & Prospective/retrospective \\
\hline 4c & IS maintenance or ISW control group yes/no \\
\hline 4d & IS drug(s) \\
\hline $4 e$ & Pre-ISW biopsy \\
\hline $4 f$ & ISW schedule \\
\hline $4 \mathrm{~g}$ & Method(s) for assessing OT \\
\hline $4 h$ & Duration of follow-up \\
\hline 5 & Study population \\
\hline $\mathbf{5 a}$ & Paediatric/adult/mixed \\
\hline $5 b$ & DDLT/LDLT/mixed \\
\hline $5 c$ & Recipient age at LT \\
\hline $5 d$ & Liver disease aetiology \\
\hline $5 e$ & Viral status during ISW \\
\hline $5 f$ & Time from LT to ISW \\
\hline $5 g$ & Reasons for ISW elective/non-elective \\
\hline 6 & Clinical parameters predicting OT \\
\hline $6 a$ & Numerical evidence for positive associations \\
\hline 7 & Biomarkers predicting OT \\
\hline $7 a$ & Numerical evidence for positive associations \\
\hline 8 & Clinical parameters not predicting OT \\
\hline 9 & Biomarkers not predicting OT \\
\hline 10 & Total number of patients that are included in the prognostic analyses \\
\hline 11 & Incidence of OT \\
\hline 12 & Number of graft losses \\
\hline
\end{tabular}


[ordered by articles, abstracts, and registry entries and by first-author names]

\begin{tabular}{|c|c|}
\hline Study (Article) & Reason for exclusion \\
\hline Aini $2014^{1}$ & Wrong Study Design \\
\hline Aini $2012^{2}$ & Wrong Study Design \\
\hline Assy $2007^{3}$ & $\begin{array}{l}\text { Incomplete Reporting (unclear how } 11 \text { non-rejection patients relate to } 2 \\
\text { OT patients) }\end{array}$ \\
\hline Bourdeaux $2013^{4}$ & Wrong Intervention (IS minimization) \\
\hline Castellaneta $2011^{5}$ & No pre-ISW Assessment \\
\hline Craciun $2007^{6}$ & Wrong Intervention (immunomodulation with stem cells) \\
\hline Devlin $1998^{7}$ & Wrong Population (> 20\% viral/autoimmune) \\
\hline Duizendstra $2019^{8}$ & Wrong Study Design (no non-OT group) \\
\hline Feng $2017^{9}$ & Wrong Study Design (no non-OT group) \\
\hline Geng $2017^{10}$ & Incomplete Reporting (non-OT group incompletely described) \\
\hline Geng $2018^{11}$ & Wrong Study Design (no ISW study) \\
\hline Girlanda $2005^{12}$ & Wrong Population ( $>20 \%$ viral/autoimmune) \\
\hline Girnita $2010^{13}$ & No pre-ISW Assessment \\
\hline Haarer $2016^{14}$ & Wrong Study Design (no ISW study) \\
\hline Hsu $2007^{15}$ & Wrong Study Design (no ISW study) \\
\hline Inomata $1999^{16}$ & $\begin{array}{l}\text { LANGUAGE (cases likely correspond to the } 26 \text { elective cases in included } \\
\text { Takatsuki report) }\end{array}$ \\
\hline Jhun $2018^{17}$ & No Prognostic Marker Reported \\
\hline Jucaud $2019^{18}$ & Wrong Population (> 20\% viral/autoimmune) \\
\hline Kasahara $2002^{19}$ & Wrong Study Design (no ISW study) \\
\hline Kawasaki $2007^{20}$ & No pre-ISW Assessment \\
\hline Koshiba $2007^{21}$ & No pre-ISW Assessment \\
\hline Koshiba $2015^{22}$ & Wrong Study Design (book chapter) \\
\hline Lau $2016^{23}$ & No pre-ISW Assessment \\
\hline Lee $2009^{24}$ & Wrong Study Design (no non-OT group) \\
\hline Levine $2017^{25}$ & Wrong Study Design (editorial) \\
\hline Li $2012^{26}$ & No pre-ISW Assessment \\
\hline Li $2008^{27}$ & No pre-ISW Assessment \\
\hline Li $2004^{28}$ & No pre-ISW Assessment \\
\hline Manez $1994^{29}$ & Wrong Intervention (temporary ISW) \\
\hline Manzia $2018^{30}$ & No Prognostic Marker Reported \\
\hline Martínez-Llordella $2008^{31}$ & No pre-ISW Assessment \\
\hline Martinez-Llordella $2007^{32}$ & No pre-ISW Assessment \\
\hline Mazariegos $1997^{33}$ & Wrong Population (> 20\% viral/autoimmune) \\
\hline Mazariegos $1997^{34}$ & Wrong Population (> 20\% viral/autoimmune) \\
\hline Mazariegos $2007^{35}$ & No pre-ISW Assessment \\
\hline Mazariegos $2005^{36}$ & No pre-ISW Assessment \\
\hline Mazariegos $2003^{37}$ & No pre-ISW Assessment \\
\hline Miura $2016^{38}$ & Wrong Study Design (no non-OT group) \\
\hline
\end{tabular}




\begin{tabular}{|c|c|}
\hline Ohe $2014^{39}$ & Wrong Study Design (classified according to fibrosis grade, not OT) \\
\hline Oike $2002^{40}$ & Incomplete Reporting \\
\hline Perito $2015^{41}$ & Wrong Outcome (post-transplant metabolic syndrome parameters) \\
\hline Picascia $2012^{42}$ & No Prognostic Marker Reported \\
\hline Ramos $1995^{43}$ & Wrong Population (> 20\% viral/autoimmune) \\
\hline Reding $2005^{44}$ & Wrong Intervention (no ISW) \\
\hline Reyes $1993^{45}$ & Wrong Study Design (no non-OT group) \\
\hline Savage $2020^{46}$ & No Prognostic Marker Reported \\
\hline Scheenstra $2006^{47}$ & Wrong Intervention (no complete ISW) \\
\hline Shaked $2019^{48}$ & Wrong Population (> 20\% viral/autoimmune) \\
\hline Shaked $2017^{49}$ & Wrong Study Design (focus on rejection) \\
\hline Shin $2013^{50}$ & Wrong Study Design (no non-OT group) \\
\hline Takatsuki $2001^{51}$ & No pre-ISW Assessment \\
\hline Taubert $2016^{52}$ & No Prognostic Marker Reported \\
\hline Tokita $2008^{53}$ & No pre-ISW Assessment \\
\hline Tryphonopoulos $2010^{54}$ & Wrong Population (> 20\% viral/autoimmune) \\
\hline Varela-Fascinetto $1997^{55}$ & Wrong Intervention (IS minimization) \\
\hline Wong $1998^{56}$ & Wrong Population (> 20\% viral/autoimmune) \\
\hline Wozniak $2015^{57}$ & No pre-ISW Assessment \\
\hline Yoshida $1998^{58}$ & Wrong Study Design (comment) \\
\hline Yoshitomi $2009^{59}$ & Wrong Study Design (no non-OT group) \\
\hline Zarkhin $2010^{60}$ & No pre-ISW Assessment \\
\hline Zhao $2013^{61}$ & No pre-ISW Assessment \\
\hline Study (Abstract) & Reason for exclusion \\
\hline Benitez $2009^{62}$ & Published as Article \\
\hline Benitez $2010^{63}$ & Published as Article \\
\hline Benitez $2010^{64}$ & Published as Article \\
\hline Bohne $2011^{65}$ & Published as Article \\
\hline Bohne $2010^{66}$ & Published as Article \\
\hline Bourdeaux $2010^{67}$ & Published as Article \\
\hline Castellaneta $2010^{68}$ & Published as Article \\
\hline Castellaneta $2010^{69}$ & Published as Article \\
\hline Castellaneta $2010^{70}$ & Published as Article \\
\hline Celik 2016 & Wrong Study Design \\
\hline Danger $2014^{72}$ & Published as Article \\
\hline Danger $2014^{73}$ & Published as Article \\
\hline De La Garza $2013^{74}$ & Published as Article \\
\hline Demetris $2010^{75}$ & Published as Article \\
\hline Ekong $2009^{76}$ & Wrong Intervention \\
\hline Feng $2016^{77}$ & Published as Article \\
\hline Feng $2016^{78}$ & Published as Article \\
\hline Feng $2014^{79}$ & Published as Article \\
\hline Feng $2009^{80}$ & Published as Article \\
\hline Feng $2011^{81}$ & Published as Article \\
\hline
\end{tabular}




\begin{tabular}{|c|c|}
\hline Feng $2011^{82}$ & Published as Article \\
\hline Fens $2011^{83}$ & Published as Article \\
\hline García De La Garza $2013^{84}$ & Published as Article \\
\hline Girnita $2010^{85}$ & Published as Article \\
\hline Herrero $2012^{86}$ & Published as Article \\
\hline Jucaud $2018^{87}$ & Published as Article \\
\hline Jucaud $2018^{88}$ & Published as Article \\
\hline Kawagishi $2014^{89}$ & Wrong Study Design \\
\hline Kim $2015^{90}$ & Published as Article \\
\hline Kim $2017^{91}$ & Wrong Study Design \\
\hline $\operatorname{Kim} 2017^{92}$ & Wrong Study Design \\
\hline Laue $2019^{93}$ & Wrong Study Design \\
\hline Levitsky $2017^{94}$ & Published as Article \\
\hline Levitsky $2016^{95}$ & Published as Article \\
\hline Li $2010^{96}$ & Published as Article \\
\hline Li $2010^{97}$ & Published as Article \\
\hline Li $2011^{98}$ & Published as Article \\
\hline Manzia $2014^{99}$ & Published as Article \\
\hline Manzia $2013^{100}$ & Wrong Study Design \\
\hline Manzia $2018^{101}$ & Published as Article \\
\hline Manzia $2012^{102}$ & Wrong Population \\
\hline Manzia $2015^{103}$ & Published as Article \\
\hline Manzia $2015^{104}$ & Published as Article \\
\hline Manzia $2010^{105}$ & Published as Article \\
\hline Manzia $2010^{106}$ & Published as Article \\
\hline Manzia $2010^{107}$ & Published as Article \\
\hline Nafady Hego $2010^{108}$ & Published as Article \\
\hline Nafady Hego $2010^{109}$ & Published as Article \\
\hline Nafady-Hego $2011^{110}$ & Published as Article \\
\hline Nafady-Hego $2012^{111}$ & Published as Article \\
\hline Ohe $2010^{112}$ & Wrong Intervention \\
\hline Ohe $2010^{113}$ & Wrong Intervention \\
\hline Reding $2009^{114}$ & Published as Article \\
\hline Scapa $2018^{115}$ & Wrong Study Design \\
\hline Schulz-Juergensen $2010^{116}$ & Wrong Study Design \\
\hline Shaked $2014^{117}$ & Published as Article \\
\hline Shaked $2016^{118}$ & Published as Article \\
\hline Shaked $2012^{119}$ & Published as Article \\
\hline Shaked $2012^{120}$ & Published as Article \\
\hline Shaked $2011^{121}$ & Published as Article \\
\hline Soyama $2012^{122}$ & Wrong Intervention \\
\hline Takatsuki $2011^{123}$ & Wrong Intervention \\
\hline Takatsuki $2017^{124}$ & Wrong Intervention \\
\hline Takatsuki $2013^{125}$ & Wrong Intervention \\
\hline Taubert $2014^{126}$ & Published as Article \\
\hline Taubert $2015^{127}$ & Published as Article \\
\hline
\end{tabular}




\begin{tabular}{|c|c|}
\hline Teisseyre $2012^{128}$ & Wrong Study Design \\
\hline Teisseyre $2014^{129}$ & Wrong Study Design \\
\hline Toti $2013^{130}$ & Wrong Study Design \\
\hline Tryphonopoulos $2010^{131}$ & Published as Article \\
\hline Tryphonopoulos $2010^{132}$ & Published as Article \\
\hline Tryphonopoulos $2012^{133}$ & Wrong Study Design \\
\hline Uchida $2012^{134}$ & Published as Article \\
\hline Wozniak $2010^{135}$ & Published as Article \\
\hline Yoshitoshi $2012^{136}$ & Wrong Study Design \\
\hline Yoshizawa $2018^{137}$ & Wrong Study Design \\
\hline Yoshizawa $2017^{138}$ & Wrong Study Design \\
\hline Zarkhin $2010^{139}$ & Published as Article \\
\hline Zhao $2012^{140}$ & Published as Article \\
\hline Study (Trial Entries) & Reason for exclusion \\
\hline NCT00135694 & Published as Article \\
\hline NCT00320606 & Published as Article \\
\hline ISRCTN16781831 & Wrong Intervention \\
\hline NCT00647283 & Published as Article \\
\hline NCT01034345 & Wrong Intervention \\
\hline NCT01198314 & Published as Article \\
\hline NCT01444079 & Wrong Intervention \\
\hline NCT01678937 & Wrong Intervention \\
\hline ISRCTN42322435 & Wrong Population \\
\hline NCT01638559 & Published as Article \\
\hline NCT02062944 & Published as Article \\
\hline ISRCTN15775356 & Published as Article \\
\hline
\end{tabular}




\section{References}

1. Aini W, Miyagawa-Hayashino A, Ozeki M, et al. Accelerated telomere reduction and hepatocyte senescence in tolerated human liver allografts. Transplant Immunology 2014; 31(2): 55-9.

2. Aini W, Miyagawa-Hayashino A, Tsuruyama T, et al. Telomere shortening and karyotypic alterations in hepatocytes in long-term transplanted human liver allografts. Transplant International 2012; 25(9): 956-66.

3. Assy N, Adams PC, Myers P, et al. Randomized controlled trial of total immunosuppression withdrawal in liver transplant recipients: Role of ursodeoxycholic acid. Transplantation 2007; 83(12): 1571-6.

4. Bourdeaux $C$, Pire $A$, Janssen $M$, et al. Prope tolerance after pediatric liver transplantation. Pediatric Transplantation 2013; 17(1): 59-64.

5. Castellaneta A, Mazariegos GV, Nayyar N, Zeevi A, Thomson AW. HLA-G level on monocytoid dendritic cells correlates with regulatory T-cell Foxp3 expression in liver transplant tolerance. Transplantation 2011; 91(10): 1132-40.

6. Craciun L, Stordeur P, Troisi R, et al. A Rapid Test of Alloreactivity Based on Interleukin-2 mRNA Expression Might Identify Liver Transplant Recipients With Donor-Specific Hyporesponsiveness. Transplantation Proceedings 2007; 39(8): 2665-7.

7. Devlin J, Doherty D, Wong T, Donaldson P, Portmann B, Williams R. Defining the outcome of immunosuppression withdrawal after liver transplantation. Hepatology 1998; 27(4): 926-33.

8. Duizendstra AA, de Knegt RJ, Betjes MGH, et al. Immunosuppressive drug withdrawal late after liver transplantation improves the lipid profile and reduces infections. European journal of gastroenterology \& hepatology 2019.

9. Feng S, Demetris AJ, Spain KM, et al. Five-year histological and serological follow-up of operationally tolerant pediatric liver transplant recipients enrolled in WISP-R. Hepatology 2017; 65(2): 647-60.

10. Geng L, Huang JJ, Lin BY, Chen TC, Shen T, Zheng SS. Characteristics of recipients with complete immunosuppressant withdrawal after adult liver transplantation. Hepatobiliary and Pancreatic Diseases International 2017; 16(4): 437-9.

11. Geng L, Liu J, Huang J, et al. A high frequency of CD8+CD28-T-suppressor cells contributes to maintaining stable graft function and reducing immunosuppressant dosage after liver transplantation. International Journal of Medical Sciences 2018; 15(9): 892-9.

12. Girlanda R, Rela M, Williams R, O'Grady JG, Heaton ND. Long-term outcome of immunosuppression withdrawal after liver transplantation. Transplantation Proceedings 2005; 37(4): 1708-9.

13. Girnita A, Mazariegos GV, Castellaneta A, et al. Liver transplant recipients weaned off immunosuppression lack circulating donor-specific antibodies. Human Immunology 2010; 71(3): 2746.

14. Haarer J, Riquelme P, Hoffmann P, et al. Early Enrichment and Restitution of the Peripheral Blood Treg Pool Is Associated With Rejection-Free Stable Immunosuppression After Liver Transplantation. Transplantation 2016; 100(7): e39-40.

15. Hsu LW, Goto S, Nakano T, et al. Immunosuppressive activity of serum taken from a liver transplant recipient after withdrawal of immunosuppressants. Transplant Immunology 2007; 17(2): 137-46.

16. Inomata $\mathrm{Y}$, Takatsuki $\mathrm{M}$, Uemoto $\mathrm{S}$, Tanaka K. Weaning of immunosuppression in livingrelated liver transplantation. Nihon Rinshō Men'eki Gakkai kaishi = Japanese journal of clinical immunology 1999; 22(6): 431-5.

17. Jhun J, Lee SH, Lee SK, et al. Serial monitoring of immune markers being represented regulatory $\mathrm{T}$ cell/T helper 17 cell ratio: Indicating tolerance for tapering immunosuppression after liver transplantation. Frontiers in Immunology 2018; 9(MAR).

18. Jucaud V, Shaked A, DesMarais M, et al. Prevalence and Impact of De Novo Donor-Specific Antibodies During a Multicenter Immunosuppression Withdrawal Trial in Adult Liver Transplant Recipients. Hepatology 2019; 69(3): 1273-86. 
19. Kasahara M, Kiuchi T, Uryuhara K, et al. Role of HLA compatibility in pediatric living-related liver transplantation. Transplantation 2002; 74(8): 1175-80.

20. Kawasaki M, Iwasaki M, Koshiba T, et al. Gene expression profile analysis of the peripheral blood mononuclear cells from tolerant living-donor liver transplant recipients. Int Surg 2007; 92(5): 276-86.

21. Koshiba T, Li Y, Takemura M, et al. Clinical, immunological, and pathological aspects of operational tolerance after pediatric living-donor liver transplantation. Transplant Immunology 2007; 17(2): 94-7.

22. Koshiba T, Li Y, Zhao X, Ohe H, Nafady-Hego H, Bishop GA. Implication of VôiyôT Cells in Operational Tolerance after Human Liver Transplantation. Advances in Medicine and Biology: Nova Science Publishers, Inc.; 2015: 113-27.

23. Lau AH, Vitalone MJ, Haas $\mathrm{K}$, et al. Mass cytometry reveals a distinct immunoprofile of operational tolerance in pediatric liver transplantation. Pediatric Transplantation 2016; 20(8): 107280.

24. Lee JH, Lee SK, Lee $\mathrm{HJ}$, et al. Withdrawal of immunosuppression in pediatric liver transplant recipients in Korea. Yonsei Medical Journal 2009; 50(6): 784-8.

25. Levine MH, Akimova T, Murken DR, Hancock WW. Regulatory T cell signatures in liver transplant recipients successfully weaned from immunosuppression: Getting from here to there. Liver Transplantation 2017; 23(7): 875-7.

26. Li L, Wozniak L, Rodder S, et al. A common peripheral blood gene set for diagnosis of operational tolerance in pediatric and adult liver transplantation. American Journal of Transplantation 2012; 12(5): 1218-28.

27. Li Y, Zhao X, Cheng D, et al. The presence of Foxp3 expressing T cells within grafts of tolerant human liver transplant recipients. Transplantation 2008; 86(12): 1837-43.

28. Li Y, Koshiba T, Yoshizawa A, et al. Analyses of peripheral blood mononuclear cells in operational tolerance after pediatric living donor liver transplantation. American Journal of Transplantation 2004; 4(12): 2118-25.

29. Manez $R$, Kusne $S$, Linden $P$, et al. Temporary withdrawal of immunosuppression for lifethreatening infections after liver transplantation. Transplantation 1994; 57(1): 149-51.

30. Maria Manzia T, Angelico R, Toti L, et al. Longterm Survival and Cost-Effectiveness of Immunosuppression Withdrawal After Liver Transplantation. Liver Transplantation 2018; 24(9): 1199208.

31. Martínez-Llordella M, Lozano JJ, Puig-Pey I, et al. Using transcriptional profiling to develop a diagnostic test of operational tolerance in liver transplant recipients. Journal of Clinical Investigation 2008; 118(8): 2845-57.

32. Martinez-Llordella M, Puig-Pey I, Orlando G, et al. Multiparameter immune profiling of operational tolerance in liver transplantation. American Journal of Transplantation 2007; 7(2): 309-

19.

33. Mazariegos GV, Reyes J, Marino I, Flynn B, Fung JJ, Starzl IE. Risks and benefits of weaning immunosuppression in liver transplant recipients: Long-term follow-up. Transplantation Proceedings 1997; 29(1-2): 1174-7.

34. Mazariegos GV, Reyes J, Marino IR, et al. Weaning of immunosuppression in liver transplant recipients. Transplantation 1997; 63(2): 243-9.

35. Mazariegos GV, Sindhi R, Thomson AW, Marcos A. Clinical tolerance following liver transplantation: Long term results and future prospects. Transplant Immunology 2007; 17(2): 114-9.

36. Mazariegos GV, Zahorchak AF, Reyes J, Chapman H, Zeevi A, Thomson AW. Dendritic cell subset ratio in tolerant, weaning and non-tolerant liver recipients is not affected by extent of immunosuppression. American Journal of Transplantation 2005; 5(2): 314-22.

37. Mazariegos GV, Zahorchak AF, Reyes J, et al. Dendritic cell subset ratio in peripheral blood correlates with successful withdrawal of immunosuppression in liver transplant patients. American Journal of Transplantation 2003; 3(6): 689-96.

38. Miura K, Kobayashi T, Zhang Z, et al. Study of Immune Tolerance Cases in Adult Living Donor Liver Transplantation. Transplantation Proceedings 2016; 48(4): 1119-22. 
39. Ohe H, Uchida $\mathrm{Y}$, Yoshizawa A, et al. Association of anti-human leukocyte antigen and antiangiotensin II type 1 receptor antibodies with liver allograft fibrosis after immunosuppression withdrawal. Transplantation 2014; 98(10): 1105-11.

40. Oike F, Yokoi A, Nishimura E, et al. Complete withdrawal of immunosuppression in living donor liver transplantation. Transplantation Proceedings 2002; 34(5): 1521.

41. Perito ER, Mohammad S, Rosenthal P, et al. Posttransplant metabolic syndrome in the Withdrawal of Immunosuppression in Pediatric Liver Transplant Recipients (WISP-R) pilot trial. American Journal of Transplantation 2015; 15(3): 779-85.

42. Picascia A, Grimaldi V, Napoli C. HLA match in operational tolerance after pediatric livingdonor liver transplantation. Transplant International 2012; 25(10): e106-e7.

43. Ramos HC, Reyes J, Abu-Elmagd K, et al. Weaning of immunosuppression in long-term liver transplant recipients. Transplantation 1995; 59(2): 212-7.

44. Reding R, Gras J, Bourdeaux C, et al. Stepwise minimization of the immunosuppressive therapy in pediatric liver transplantation: A conceptual approach towards operational tolerance. Acta Gastro-Enterologica Belgica 2005; 68(3): 320-2.

45. Reyes J, Zeevi A, Ramos $\mathrm{H}$, et al. Frequent achievement of a drug-free state after orthotopic liver transplantation. Transplantation Proceedings 1993; 25(6): 3315-9.

46. Savage TM, Shonts BA, Lau S, et al. Deletion of donor-reactive T cell clones after human liver transplant. American Journal of Transplantation 2020; 20(2): 538-45.

47. Scheenstra R, Torringa MLJ, Waalkens HJ, et al. Cyclosporine a withdrawal during follow-up after pediatric liver transplantation. Liver Transplantation 2006; 12(2): 240-6.

48. Shaked A, DesMarais MR, Kopetskie $\mathrm{H}$, et al. Outcomes of immunosuppression minimization and withdrawal early after liver transplantation. American Journal of Transplantation 2019; 19(5): 1397-409.

49. Shaked A, Chang BL, Barnes MR, et al. An ectopically expressed serum miRNA signature is prognostic, diagnostic, and biologically related to liver allograft rejection. Hepatology 2017; 65(1): 269-80.

50. Shin $\mathrm{M}$, Song $\mathrm{S}$, Moon $\mathrm{HH}$, et al. Characteristics of recipients who achieved spontaneous operational tolerance in adult liver transplantation. Transplantation Proceedings 2013; 45(8): 3024-7.

51. Takatsuki M, Uemoto $S$, Inomata $Y$, et al. Analysis of alloreactivity and intragraft cytokine profiles in living donor liver transplant recipients with graft acceptance. Transplant Immunology 2001; 8(4): 279-86.

52. Taubert R, Danger R, Londono MC, et al. Hepatic Infiltrates in Operational Tolerant Patients After Liver Transplantation Show Enrichment of Regulatory T Cells Before Proinflammatory Genes Are Downregulated. Am J Transplant 2016; 16(4): 1285-93.

53. Tokita D, Mazariegos GV, Zahorchak AF, et al. High PD-L1/CD86 ratio on plasmacytoid dendritic cells correlates with elevated T-regulatory cells in liver transplant tolerance.

Transplantation 2008; 85(3): 369-77.

54. Tryphonopoulos $\mathrm{P}$, Ruiz $\mathrm{P}$, Weppler $\mathrm{D}$, et al. Long-term follow-up of 23 operational tolerant liver transplant recipients. Transplantation 2010; 90(12): 1556-61.

55. Varela-Fascinetto G, Treacy SJ, Vacanti JP. Approaching operational tolerance in long-term pediatric liver transplant recipients receiving minimal immunosuppression. Transplantation Proceedings 1997; 29(1-2): 449-51.

56. Wong T, Nouri-Aria KT, Devlin J, Portmann B, Williams R. Tolerance and latent cellular rejection in long-term liver transplant recipients. Hepatology 1998; 28(2): 443-9.

57. Wozniak LJ, Hickey MJ, Venick RS, et al. Donor-specific HLA Antibodies Are Associated with Late Allograft Dysfunction after Pediatric Liver Transplantation. Transplantation 2015; 99(7): 141622.

58. Yoshida EM, Chung SW. Outcomes after complete immunosuppression withdrawal in liver transplant recipients: Rejection-free graft tolerance or late acute rejection? Canadian Journal of Gastroenterology 1998; 12(6): 387-9. 
59. Yoshitomi M, Koshiba $\mathrm{T}$, Haga $\mathrm{H}$, et al. Requirement of protocol biopsy before and after complete cessation of immunosuppression after liver transplantation. Transplantation 2009; 87(4): 606-14.

60. Zarkhin V, Talisetti A, Li L, et al. Expression of soluble HLA-G identifies favorable outcomes in liver transplant recipients. Transplantation 2010; 90(9): 1000-5.

61. Zhao $\mathrm{X}, \mathrm{Li} \mathrm{Y}, \mathrm{Ohe} \mathrm{H}$, et al. Intragraft $\mathrm{V} \delta 1 \gamma \delta \mathrm{T}$ cells with a unique T-Cell receptor are closely associated with pediatric semiallogeneic liver transplant tolerance. Transplantation 2013; 95(1): 192 202.

62. Benitez C, Lozano JJ, Martinez-Llordella M, et al. Use of transcriptional biomarkers to identify liver transplant (Table 1). Thus the transcriptome in renal allograft biopsies predicts the risk of recipients who can successfully discontinue immunosuppressive graft loss beyond the predictive value of time post transplant, renal function, and therapy. American Journal of Transplantation 2009; 9: 252.

63. Benitez CE, Lozano JJ, Martínez-Llordella M, et al. Use of transcriptional biomarkers to identify liver transplant recipients who can successfully discontinue immunosuppressive therapy. American Journal of Transplantation 2010; 10: 2-3.

64. Benitez CE, Lozano JJ, Martinez-Llordella M, et al. Use of transcriptional biomarkers to identify liver transplant recipients who can successfully discontinue immunosuppressive therapy. American Journal of Transplantation 2010; 10: 191-2.

65. Bohne F, Martinez LM, Lozano JJ, et al. Transcriptional profiling of liver grafts in spontaneous operational tolerance. American Journal of Transplantation 2011; 11: 390.

66. Bohne F, Martinez-Llordella M, Lozano JJ, et al. Transcriptional profiling of liver grafts in spontaneous operational tolerance. Transplantation 2010; 90: 13.

67. Bourdeaux $C$, Pire A, Janssens $M$, et al. Liver transplantation during infancy provides similar overall results and might enhance prope/operational tolerance in the long term. Liver Transplantation 2010; 16: S116.

68. Castellaneta A, Mazariegos G, DeVera ME, et al. Elevated hla-g and ilt4 expression on circulating dendritic cell subsets in pediatric liver transplant recipients successfully weaned off immunosuppression. Transplantation 2010; 90: 1077.

69. Castellaneta A, Mazariegos GV, DeVera M, et al. Elevated HLA-G and ILT4 expression on circulating dendritic cell subsets in pediatric liver transplant recipients successfully weaned off immunosuppression. American Journal of Transplantation 2010; 10: 1.

70. Castellaneta A, Mazariegos GV, Girnita A, et al. Liver transplant recipients successfully weaned off immunosuppression lack donor-specific anti-HLA antibodies. American Journal of Transplantation 2010; 10: 481.

71. Celik N, Ganoza A, Vyas F, et al. Long-term outcomes in pediatric live-donor liver transplantation. Transplantation 2016; 100(7): S531.

72. Danger $\mathrm{R}$, Taubert $\mathrm{R}$, Londono $\mathrm{M}$, et al. Immune-regulatory pathways are transiently activated during the spontaneous establishment of operational tolerance following liver transplantation. Transplantation 2014; 98: 25.

73. Danger $\mathrm{R}$, Taubert $\mathrm{R}$, Londoño $\mathrm{MC}$, et al. Immune-regulatory pathways are transiently activated during the spontaneous establishment of operational tolerance following liver transplantation. Liver Transplantation 2014; 20: S138.

74. De La Garza RG, Merino J, D'Avola D, et al. Evolution of lymphocyte subpopulations after immunosuppression withdrawal in tolerant liver transplant recipients. Hepatology 2013; 58(4): 1037 .

75. Demetris AJ, Ruppert K, Ekong U, Lobritto SJ, Tchao NK, Feng S. Prospective evaluation of histopathologic biopsy features that predict successful weaning and comparison to protocol followup biopsies 2 years after complete immunosuppression withdrawal in living-related pediatric liver allograft recipients. Hepatology 2010; 52: 322A-3A.

76. Ekong UD, Lokar J, Cymerman I, Alonso EM. Successful immunosuppression minimization in pediatric LT. American Journal of Transplantation 2009; 9: 254. 
77. Feng S, Bucuvalas J, Demetris A, et al. Primary outcome of iWITH: A multi-center clinical trial of complete immunosuppression withdrawal (ISW) in stable pediatric liver transplant (LT) recipients. American Journal of Transplantation 2016; 16: 269.

78. Feng S, Bucuvalas J, Demetris AJ, et al. Primary outcome of iWITH: A 12 center North American clinical trial of immunosuppression withdrawal in stable pediatric liver transplant recipients. Transplantation 2016; 100(7): S348-S9.

79. Feng S, Demetris AJ, Isse K, et al. Serum and tissue DSA subclass, stellate and endothelial phenotype monitoring in itn029st tolerant pediatric liver transplant recipients over $5+$ years of follow-up. Liver Transplantation 2014; 20: S117.

80. Feng S, Ekong U, Lobritto S, et al. ITN029ST: Immunosuppression withdrawal in pediatric recipients of parental living donor liver transplants: Preliminary results of a pilot study. American Journal of Transplantation 2009; 9: 244-5.

81. Feng $\mathrm{S}$, Ekong $\mathrm{U}$, Lobritto $\mathrm{S}$, et al. Clinical and histological predictors of operational tolerance in pediatric liver transplant recipients. American Journal of Transplantation 2011; 11: 76.

82. Feng S, Ekong $\mathrm{U}$, Lobrttto $\mathrm{S}$, et al. Clinical and histological predictors of operational tolerance in pediatric liver transplant recipients. Liver Transplantation 2011; 17: S107.

83. Fens S, Punch J, Reyes J, et al. Evolution of donor specific alloantibodies (DSA) with immunosuppression (IS) withdrawal among adult liver transplant recipients in 1TNQ3QST. Liver Transplantation 2011; 17: S85.

84. García De La Garza R, Sarobe P, Merino J, et al. Factors predictive of tolerance after liver transplantation. Journal of Hepatology 2013; 58: S231.

85. Girnita A, Mazariegos GV, Castellaneta A, et al. Liver transplant recipients successfully weaned off immunosuppression lack donor-specific anti-HLA antibodies. American Journal of Transplantation 2010; 10: 25-6.

86. Herrero JI, Sarobe $\mathrm{P}$, Merino J, et al. Factors predictive of tolerance after liver transplantation. Liver Transplantation 2012; 18: S187.

87. Jucaud V, Shaked A, DesMarais M, Sayre P, Everly M. Development of post-transplant DSA following immunosuppression minimization/withdrawal in liver transplant recipients. American Journal of Transplantation 2018; 18: 472-3.

88. Jucaud V, Shaked A, DesMarais M, Sayre P, Everly MJ. Development of post-transplant DSA in adult liver transplant patients from the ITN a-wish trial. Transplantation 2018; 102(7): S203.

89. Kawagishi N, Tokodai K, Takeda I, Miyagi S, Satoh K, Ohuchi N. Long-term results on the immunosuppression withdrawal in pediatric recipients of parental living donor liver transplants. Transplantation 2014; 98: 713.

90. Kim HY, Choi JY, Jhun JY, Cho ML, Kim DG. Prospective immunosuppression withdrawal under immunological monitoring among adult liver transplant recipients. Transplantation 2015; 99(7): 275.

91. Kim IG, Hu XG, Wang HJ, Kim BW, Hong SY. Minimization of immunosuppression after liver transplantation from the perspectives of 'de novo malignancy' and 'nephrotoxicity'. Transplant International 2017; 30: 479.

92. Kim IG, Hu XG, Wang HJ, Kim BW, Hong SY. Minimization of immunosuppression after liver transplantation from the perspectives of 'de novo malignancy' and 'nephrotoxicity'. Journal of Hepato-Biliary-Pancreatic Sciences 2017; 24: A128.

93. Laue T, Goldschmidt I, Pfister E, et al. Biomarkers for Immune function after liver transplantation in children and adolescents with standard calcineurin inhibitor-based versus reduced minimal immunosuppression. Journal of Pediatric Gastroenterology and Nutrition 2019; 68: 947-8.

94. Levitsky J, Kurian SM, Sanchez-Fueyo A, Mathew JM, Heinrichs J, Burrell BE. Blood and graft biomarkers predictive of operational tolerance in liver transplant recipients on sirolimus. Hepatology 2017; 66: 3A-4A.

95. Levitsky J, Mathew JM, Miller J, Heinrichs J, Huang X, Abecassis MM. Immunosuppression withdrawal in liver transplant recipients on sirolimus. Hepatology 2016; 64(1): $1 \mathrm{~A}$.

96. Li L, Talisetti A, Hsieh S, et al. A peripheral blood 12 gene-set for diagnosis of pediatric liver allograft tolerance. Transplantation 2010; 90: 405. 
97. Li L, Talisetti A, Hsieh S, et al. A peripheral blood 12 gene-set for diagnosis of pediatric liver allograft tolerance. American Journal of Transplantation 2010; 10: 11.

98. Li L, Wozniak L, Heish S, et al. A peripheral blood 13 gene-set for diagnosis of pediatric liver allograft tolerance. Pediatric Transplantation 2011; 15: 60.

99. Manzia T, Angelico R, Gherardi D, et al. Long-term outcome and cost effectiveness of sustained immunosuppression withdrawal after liver transplantation. Transplantation 2014; 98: 709.

100. Manzia T, Angelico R, Toti L, et al. The long term clinical outcome of liver transplant recipients who successfully withdrawn immunosuppression. American Journal of Transplantation 2013; 13: 540.

101. Manzia TM, Angelico C, lesari S, Ackenine K, Lerut J, Tisone G. Cost effectiveness of immunosuppression withdrawal after liver transplantation. Transplantation 2018; 102(7): S121.

102. Manzia TM, Angelico R, Ciano P, et al. The long term follow-up immunosuppression free state in HCV liver transplant recipients. American Journal of Transplantation 2012; 12: 197.

103. Manzia TM, Angelico R, Gherardi D, et al. Long-term outcome and cost saving of immunosuppression withdrawal after liver transplantation. Transplantation 2015; 99(7): 130.

104. Manzia TM, Angelico R, Toti L, et al. Cost saving of immunosuppression withdrawal after liver transplantation in a spending review era. Transplant International 2015; 28: 200.

105. Manzia TM, Angelico R, Toti L, et al. Predicting "operational tolerance" after liver transplantation. Transplantation 2010; 90: 34.

106. Manzia TM, Toti L, Angelico R, Sorge R, Bellini I, Anselmo A. Weaning off immunosuppression after liver transplantation: Predictive factors and short-term results. Liver Transplantation 2010; 16: S73.

107. Manzia TM, Toti L, Angelico R, et al. "Operational tolerance" in stable liver transplant recipients: Predictive factors and short-term results. American Journal of Transplantation 2010; 10: 174.

108. Nafady Hego HA, Li Y, Ohe H, Sakaguchi S, Uemoto S, Koshiba T. Critical role of naive regulatory $\mathrm{T}$ cells in operational tolerance after pediatric living-donor liver transplantation. Transplantation 2010; 90: 404.

109. Nafady Hego HA, Li Y, Sakaguchi S, Uemoto S, Koshiba T. Up regulation of FOXP3 expression in CD4+CD25+ IL-7R $\alpha$ low regulatory T cells in operational tolerance after pediatric living donor liver transplantation. Transplantation 2010; 90: 9.

110. Nafady-Hego H, Li Y, Ohe H, et al. Upregulation of FOXP3 expression of donor specific CD4+CD25++IL-7low+ regulatory $T$ cells in operationally tolerant recipients after pediatric livingdonor liver transplantation. American Journal of Transplantation 2011; 11: 392.

111. Nafady-Hego H, Li Y, Ohe H, et al. The value of CD127 to isolate and define CD4+CD25++ regulatory $\mathrm{T}$ cells in operationally tolerant recipients after pediatric liver transplantation. Transplantation 2012; 94: 481.

112. Ohe H, Egawa H, Uemoto S, Koshiba T. Prope tolerance: A more realistic approach to improve long-term outcomes for pediatric living-donor liver transplantation. American Journal of Transplantation 2010; 10: 347.

113. Ohe H, Koshiba T, Li Y, et al. Prope tolerance : A more realistic approach to improve longterm outcomes for pediatric living-donor liver transplantation. Transplantation 2010; 90: 325.

114. Reding R, Pire A, Janssen M, Brunati A, De Magnée C, Bourdeaux C. Steroid-free tacrolimusbased immunosuppression promotes long-term prope/operational tolerance in pediatric liver transplantation. Pediatric Transplantation 2009; 13: 63-4.

115. Scapa J, Wozniak L, Naini BV. Can't the liver be a little more tolerant: A clinicopathologic study of immunologic tolerant pediatric liver transplant recipients. Laboratory Investigation 2018; 98 : 648.

116. Schulz-Juergensen S, Marischen L, Wesch D, Kabelitz D, Kohl M, Burdelski M. Diagnostic value of markers of operational immune tolerance after pediatric liver transplantation. Journal of Pediatric Gastroenterology and Nutrition 2010; 50: E46-E7.

117. Shaked A, Chang B, Guettouche T, et al. Potential application of serum miRNA signature for minimization of immunosuppression and diagnosis of rejection following liver transplantation. 
American journal of transplantation Conference: 2014 world transplant congress United states 2014; 14(Supplement 3): 230.

118. Shaked A, Feng S, Punch J, et al. Early post-transplant immunosuppression (IS) withdrawalFinal outcomes of the ITN030ST AWISH Study. American Journal of Transplantation 2016; 16: 269.

119. Shaked A, Feng S, Punch J, et al. Initial outcomes of ITNO30ST: Early post-liver transplant immunosuppression withdrawal. Liver Transplantation 2012; 18: S106.

120. Shaked A, Feng S, Punch J, et al. Initial outcomes of ITNO30ST: Early post-liver transplant immunosuppression withdrawal. American Journal of Transplantation 2012; 12: 197.

121. Shaked A, Feng S, Punch J, et al. Lessons learned from the ITNO30ST immunosuppression withdrawal (ISW) trial in liver transplant recipients. Liver Transplantation 2011; 17: S84.

122. Soyama A, Takatsuki M, Muraoka I, et al. Chronological evaluation of immune status by immune function assay (immuknow) for weaning of immunosuppression in living donor liver transplantation recipient. Liver Transplantation 2012; 18: S201.

123. Takatsuki M, Eguchi S, Hidaka M, et al. Impact of peripheral blood CD4+ adenosine triphosphate activity in long-term living donor liver transplantation under weaning of immunosuppression. Liver Transplantation 2011; 17: S207.

124. Takatsuki M, Okada S, Hidaka M, et al. Elective weaning of immunosuppression in living donor liver transplant recipients. Transplant International 2017; 30: 453.

125. Takatsuki M, Soyama A, Torashima $\mathrm{Y}$, et al. Weaning of immunosuppression in living donor liver transplant recipients. Liver Transplantation 2013; 19(6): S90.

126. Taubert R, Danger R, Londono MC, et al. Operational tolerance causes a long lasting active immunoregulation within the graft. Transplant International 2014; $27: 14$.

127. Taubert R, Danger R, Londono MC, et al. Operational tolerance causes a long lasting active immunoregulation within the graft. Journal of Hepatology 2015; 62: S248-S9.

128. Teisseyre J, Markiewicz-Kijewska M, Kalicinski P, Teisseyre M. Withdrawal of immunossupresion after liver transplantation-own experience. Transplantation 2012; 94: 746.

129. Teisseyre J, Markiewicz-Kijewska M, Kalicinski P, Ismail H, Teisseyre M, Jankowska I. Immunosuppression withdrawal in children after liver transplantation-is it possible? Liver Transplantation 2014; 20: S174.

130. Toti L, Manzia TM, Angelico R, et al. The Tor Vergata weaning of immunosuppression in liver transplant patients: The long-term follow-up. Liver Transplantation 2013; 19(6): S208.

131. Tryphonopoulos $\mathrm{P}$, Ruiz $\mathrm{P}, \mathrm{Weppler} \mathrm{D}$, et al. Long term follow up of 22 operational tolerant liver transplant recipients. Transplantation 2010; 90: 33.

132. Tryphonopoulos $\mathrm{P}$, Ruiz $\mathrm{P}$, Weppler $\mathrm{D}$, et al. Clinical outcome of liver transplant recipients after successful immunosuppression withdrawal. American Journal of Transplantation 2010; 10: 2. 133. Tryphonopoulos PT, Ruiz P, Weppler D, et al. Long term follow up of 30 operationally tolerant liver transplant recipients. Transplantation 2012; 94: 130.

134. Uchida $\mathrm{Y}$, Ohe $\mathrm{H}$, Yoshizawa $\mathrm{A}$, et al. Is it possible to wean immunosuppression in pediatric liver transplantation? The problem of long-term management. Liver Transplantation 2012; 18: S82S3.

135. Wozniak LJ, Li L, Hsieh S, et al. Validation of a transcriptional profile of tolerance in pediatric liver transplant recipients. Transplantation 2010; 90: 402.

136. Yoshitoshi EY, Yoshizawa A, Kaneshiro M, Takada N, Okamoto S, Uemoto S. The long-term impact of immunosupression weaning in renal function of children after living-donor liver transplantation. Transplantation 2012; 94: 223.

137. Yoshizawa A, Kaneshiro M, Uebayashi E, et al. The analysis of pathological and serological findings in the long-term pediatric liver transplantation: The achievement of clinical operational tolerance. Transplantation 2018; 102(7): S896.

138. Yoshizawa A, Kaneshiro M, Ueda D, et al. The status of immunosuppressive therapy for the outpatients after pediatric liver transplantion: The achievement of clinical operational tolerance. Pediatric Transplantation 2017; 21: 21.

139. Zarkhin V, Talisetti A, Cox K, Hurwitz M, Esquivel C, Sarwal M. HLA-G: A novel serological marker for liver transplant tolerance. American Journal of Transplantation 2010; 10: 178. 
140. Zhao X, Li Y, Waki K, et al. Propagation of $v \delta 1$ $\delta \delta$ T cells and their skewed T cell receptor within accepted grafts after pediatric semi-allogeneic liver transplantation. Transplantation 2012; 94: 56. 
Table S3: Characteristics of included studies: Chronological overview of reports

\begin{tabular}{|c|c|c|c|c|c|c|c|c|c|c|c|c|c|c|c|}
\hline $\begin{array}{l}\text { First Author } \\
\text { Year (Country) }\end{array}$ & $\begin{array}{l}\text { Study ID } \\
\text { (Cohort } \\
\text { Overlap) }\end{array}$ & $\begin{array}{l}\text { Study } \\
\text { Population }\end{array}$ & Study Design & $\begin{array}{l}\text { Control } \\
\text { Group } \\
\text { (IS } \\
\text { Mainten } \\
\text { ance or } \\
\text { Weaning } \\
\text {, }\end{array}$ & $\begin{array}{l}D D / L D \\
L T\end{array}$ & $\begin{array}{l}\text { Mean/median } \\
\text { Age at } \angle T \\
\text { (range)/years }\end{array}$ & $\begin{array}{l}\text { Liver Disease } \\
\text { Etiology }\end{array}$ & $\begin{array}{l}\text { Viral Status } \\
\text { during ISW }\end{array}$ & $\begin{array}{l}\text { IS Drug(s) } \\
\text { at ISW } \\
\text { Enrollment }\end{array}$ & $\begin{array}{l}\text { Reason(s) } \\
\text { for ISW }\end{array}$ & $\begin{array}{l}\text { Pre-ISW } \\
\text { Biopsy }\end{array}$ & ISW Schedule & $\begin{array}{l}\text { Assessment } \\
\text { of OT }\end{array}$ & $\begin{array}{l}\text { Mean/median } \\
\text { (range) } \\
\text { FU/months }\end{array}$ & $\begin{array}{l}\text { \# Patients } \\
\text { Included in } \\
\text { Prognostic } \\
\text { Analyses } \\
\text { (Total) }\end{array}$ \\
\hline $\begin{array}{r}\text { Takatsuki } 2001 \\
\text { (Japan) }\end{array}$ & "Kyoto" & Pediatric & $\begin{array}{l}\text { Monocenter } \\
\text { Retrospective }\end{array}$ & No & LD & $\begin{array}{l}N R / 1.1(0.1- \\
15.2)\end{array}$ & $\begin{array}{l}\text { non- } \\
\text { autoimmune }\end{array}$ & $\begin{array}{l}\text { no patients } \\
\text { with viral } \\
\text { liver } \\
\text { disease } \\
\text { etiology }\end{array}$ & $\mathrm{TAC}+\mathrm{CS}$ & $\begin{array}{l}\text { elective } \\
\text { non- } \\
\text { elective }\end{array}$ & NR & $\begin{array}{l}\text { elective: doses } \\
\text { gradually } \\
\text { decreased over } \\
\text { 3-6 mo } \\
\text { non-elective: } \\
\text { individually, } \\
\text { depending on } \\
\text { clinical course } \\
\text { and indication }\end{array}$ & LFT & $\begin{array}{l}N R / 23.5 \text { (3 - } \\
69)[O T]\end{array}$ & 63 \\
\hline $\begin{array}{r}\text { Pons } 2003 \\
\text { (Spain) }\end{array}$ & "Murcia 1" & Adult & $\begin{array}{l}\text { Monocenter } \\
\text { Prospective }\end{array}$ & No & DD & $\begin{array}{l}53 / 56(35- \\
65)^{3}\end{array}$ & $\begin{array}{l}\text { non- } \\
\text { autoimmune }\end{array}$ & $\begin{array}{l}\text { no patients } \\
\text { with viral } \\
\text { liver } \\
\text { disease } \\
\text { etiology }\end{array}$ & $\mathrm{Cs} A+\mathrm{CS}$ & elective & Yes & $\begin{array}{l}10 \% \\
\text { decrements at } \\
1 \text { mo intervals, } \\
\text { followed by } \\
\text { prednisone } \\
\text { withdrawal } \\
\text { (over } 2 \mathrm{mo} \text { ) }\end{array}$ & $\begin{array}{l}\text { LFT } \\
\text { Biopsy }\end{array}$ & (17 - 24) [OT] & 9 \\
\hline $\begin{array}{r}\text { Pons } 2008 \\
\text { (Spain) }\end{array}$ & "Murcia 1" & Adult & $\begin{array}{l}\text { Monocenter } \\
\text { Prospective }\end{array}$ & Yes & DD & $48 / \mathrm{NR} \pm 10^{3}$ & $\begin{array}{l}\text { non- } \\
\text { autoimmune }\end{array}$ & $\begin{array}{l}\text { no patients } \\
\text { with viral } \\
\text { liver } \\
\text { disease } \\
\text { etiology }\end{array}$ & CsA & elective & Yes & $\begin{array}{l}10 \% \\
\text { decrements at } \\
1 \text { mo intervals, } \\
\text { followed by } \\
\text { prednisone } \\
\text { withdrawal } \\
\text { (over } 2 \mathrm{mo} \text { ) }\end{array}$ & $\begin{array}{l}\text { LFT } \\
\text { Biopsy }\end{array}$ & $(10$ - 30) [OT] & 12 \\
\hline $\begin{array}{r}\text { Pons } 2009 \\
\text { (Spain) }\end{array}$ & "Murcia 1" & Adult & $\begin{array}{l}\text { Monocenter } \\
\text { Prospective }\end{array}$ & No & DD & $\begin{array}{l}48 / N R \pm 9.5 \\
(31-62)\end{array}$ & $\begin{array}{l}\text { non- } \\
\text { autoimmune }\end{array}$ & $\begin{array}{l}\text { no patients } \\
\text { with viral } \\
\text { liver } \\
\text { disease } \\
\text { etiology }\end{array}$ & $\begin{array}{l}\text { CsA } \\
\text { CsA+CS } \\
\text { Other }\end{array}$ & $\begin{array}{l}\text { elective } \\
\text { non- } \\
\text { elective }\end{array}$ & $\begin{array}{l}\text { Yes (only } \\
\text { elective } \\
\text { cases) }\end{array}$ & $\begin{array}{l}\text { elective: } 10 \% \\
\text { decrements at } \\
1 \text { mo intervals, } \\
\text { followed by } \\
\text { prednisone } \\
\text { withdrawal } \\
\text { (over } 2 \text { mo) } \\
\text { non-elective: } \\
\text { over } 1-6 \text { mo }\end{array}$ & $\begin{array}{l}\text { LFT } \\
\text { Biopsy }\end{array}$ & $53 / 48(10-132)$ & 20 \\
\hline $\begin{array}{l}\text { Millán } 2010 \\
\text { (Spain) }^{1}\end{array}$ & $\begin{array}{l}\text { "Barcelona/R } \\
\text { ome/Leuven" }\end{array}$ & Adult & $\begin{array}{l}\text { Monocenter } \\
\text { Prospective }\end{array}$ & No & DD & $\begin{array}{l}45 / 47(19- \\
63)\end{array}$ & $\begin{array}{l}\text { non- } \\
\text { autoimmune }\end{array}$ & $\begin{array}{l}<20 \% \\
\text { replicative } \\
\text { viral } \\
\text { disease }\end{array}$ & $\begin{array}{l}\text { TAC } \\
\text { CSA } \\
\text { MMF } \\
\text { Other }\end{array}$ & elective & NR & $\begin{array}{l}\text { decreased in } \\
\text { monthly } \\
\text { intervals, } \\
\text { complete } \\
\text { discontinuation } \\
\text { after 6-9 mo }\end{array}$ & NR & $>12$ & 24 \\
\hline $\begin{array}{l}\text { Nafady-Hego } \\
2010 \text { (Japan) }\end{array}$ & "Kyoto" & Pediatric & $\begin{array}{l}\text { Monocenter } \\
\text { Retrospective }\end{array}$ & Yes & LD & $\begin{array}{l}{[17 / \mathrm{NR} \text { (7 - }} \\
28)] \text { OT } \\
{[11 / \mathrm{NR}(5 \text { - }} \\
22)] \text { nonOT }\end{array}$ & $\begin{array}{l}\text { non- } \\
\text { autoimmune }\end{array}$ & $\begin{array}{l}\text { no patients } \\
\text { with viral } \\
\text { liver } \\
\text { disease } \\
\text { etiology }\end{array}$ & $\begin{array}{l}\text { TAC } \\
\text { Other }\end{array}$ & $\begin{array}{l}\text { elective } \\
\text { non- } \\
\text { elective }\end{array}$ & NR & $\mathrm{NR}$ & LFT & $88 / N R \pm 34$ & 42 \\
\hline
\end{tabular}




\begin{tabular}{|c|c|c|c|c|c|c|c|c|c|c|c|c|c|c|c|}
\hline $\begin{array}{r}\text { Talisetti } 2010 \\
\text { (USA) }\end{array}$ & "Stanford" & Pediatric & $\begin{array}{l}\text { Multicenter } \\
\text { Retrospective }\end{array}$ & Yes & both & $\begin{array}{l}\text { 1.4/NR (0.3 - } \\
4.9) \text { [OT] } \\
3.4 / \mathrm{NR}(0.3- \\
\text { 16) [nonOT] }\end{array}$ & $\begin{array}{l}\text { non- } \\
\text { autoimmune }\end{array}$ & $\begin{array}{l}\text { no patients } \\
\text { with viral } \\
\text { liver } \\
\text { disease } \\
\text { etiology }\end{array}$ & $\begin{array}{l}\text { TAC } \\
\text { CsA }\end{array}$ & $\begin{array}{l}\text { non- } \\
\text { elective } \\
\text { NR }\end{array}$ & NR & NR & LFT & $\begin{array}{l}91 / \text { NR (24 - } \\
\text { 145) [OT] }\end{array}$ & 62 \\
\hline $\begin{array}{r}\text { Waki } 2011 \\
\text { (Japan) }\end{array}$ & "Tochigi" & Pediatric & $\begin{array}{l}\text { Monocenter } \\
\text { Retrospective }\end{array}$ & No & LD & $\begin{array}{l}2.1 / \mathrm{NR} \pm 2.6 \\
{[\mathrm{OT}]} \\
3.3 / \mathrm{NR} \pm 4.1 \\
{[\text { nonOT] }}\end{array}$ & $\begin{array}{l}\text { non- } \\
\text { autoimmune }\end{array}$ & $\begin{array}{l}\text { no patients } \\
\text { with viral } \\
\text { liver } \\
\text { disease } \\
\text { etiology }\end{array}$ & $\begin{array}{l}\mathrm{TAC}+\mathrm{CS} \\
\mathrm{CSA}+\mathrm{CS}\end{array}$ & elective & NR & $\begin{array}{l}\text { gradual } \\
\text { reduction over } \\
2 \mathrm{yr}\end{array}$ & LFT & NR & 52 \\
\hline $\begin{array}{r}\text { Bohne } 2012 \\
\text { (Spain, Italy, } \\
\text { Belgium) }\end{array}$ & $\begin{array}{l}\text { "Barcelona/R } \\
\text { ome/Leuven" } \\
\text { NCT00647283 }\end{array}$ & Adult & $\begin{array}{l}\text { Multicenter } \\
\text { Prospective }\end{array}$ & Yes & NR & $\begin{array}{l}\text { [NR/62 (27- } \\
73)[\mathrm{OT}] \\
\text { NR/51 (25- } \\
71) \text { [nonOT]] }\end{array}$ & $\begin{array}{l}\text { non- } \\
\text { autoimmune }\end{array}$ & $\begin{array}{l}<20 \% \\
\text { replicative } \\
\text { viral } \\
\text { disease }\end{array}$ & $\begin{array}{l}\text { TAC } \\
\text { CSA } \\
\text { MMF } \\
\text { AZA } \\
\text { "No-CNI" }\end{array}$ & elective & Yes & $\begin{array}{l}\text { Over } 6 \text { to } 9 \\
\text { months: first } \\
\text { MMF over } 3 \\
\text { months then } \\
\text { CNI } 1 / 4 \text { to } 1 / 2 \\
\text { every } 3 \text { weeks }\end{array}$ & $\begin{array}{l}\text { LFT } \\
\text { Biopsy }\end{array}$ & NR & 75 \\
\hline $\begin{array}{r}\text { Feng } 2012 \\
\text { (USA) }\end{array}$ & $\begin{array}{l}\text { "WISP-R" } \\
\text { NCT00320606 }\end{array}$ & Pediatric & $\begin{array}{l}\text { Multicenter } \\
\text { Prospective }\end{array}$ & No & LD & $\begin{array}{l}1.0 / 0.6(0.3- \\
7.5)\end{array}$ & $\begin{array}{l}\text { non- } \\
\text { autoimmune }\end{array}$ & $\begin{array}{l}\text { no patients } \\
\text { with viral } \\
\text { liver } \\
\text { disease } \\
\text { etiology }\end{array}$ & $\begin{array}{l}\text { TAC } \\
\text { CsA }\end{array}$ & elective & Yes & $\begin{array}{l}\text { stepwise over }> \\
36 \text { wk } \\
\text { (decrease every } \\
4 \text { to } 8 w k \text { ) }\end{array}$ & $\begin{array}{l}\text { LFT } \\
\text { Biopsy }\end{array}$ & $\begin{array}{l}\mathrm{NR} / 32.9 \text { (IQR } \\
1.0-49.9)\end{array}$ & 20 \\
\hline $\begin{array}{r}\text { Ohe } 2012 \\
\text { (Japan) }\end{array}$ & "Kyoto" & Pediatric & $\begin{array}{l}\text { Monocenter } \\
\text { Retrospective }\end{array}$ & Yes & LD & $\begin{array}{l}2.9 / \mathrm{NR} \pm 3.7 \\
{[\mathrm{OT}]} \\
2.1 / \mathrm{NR} \pm 2.9 \\
{[\text { nonOT] }}\end{array}$ & NR & NR & $\begin{array}{l}\text { TAC } \\
\text { CSA }\end{array}$ & $\begin{array}{l}\text { elective } \\
\text { non- } \\
\text { elective }\end{array}$ & NR & $\begin{array}{l}\text { elective: bid, } \\
\text { qd, } 4 \text { times a } \\
\text { wk, twice a wk, } \\
\text { once a wk, } \\
\text { twice a mo, } \\
\text { once a mo, and } \\
\text { stop ( } 3-6 \text { mo } \\
\text { interval } \\
\text { between each } \\
\text { step) }\end{array}$ & $\begin{array}{l}\text { LFT } \\
\text { Biopsy }\end{array}$ & NR & 134 \\
\hline $\begin{array}{r}\text { Benitez } 2013 \\
\text { (Spain, Italy, } \\
\text { Belgium) }\end{array}$ & $\begin{array}{l}\text { "Barcelona/R } \\
\text { ome/Leuven" } \\
\text { NCT00647283 }\end{array}$ & Adult & $\begin{array}{l}\text { Multicenter } \\
\text { Prospective }\end{array}$ & No & NR & $47 / N R \pm 10$ & $\begin{array}{l}\text { non- } \\
\text { autoimmune }\end{array}$ & $\begin{array}{l}<20 \% \\
\text { replicative } \\
\text { viral } \\
\text { disease }\end{array}$ & $\begin{array}{l}\text { TAC } \\
\text { CsA } \\
\text { MMF } \\
\text { AZA } \\
\text { Other }\end{array}$ & elective & Yes & $\begin{array}{l}\text { Over } 6 \text { to } 9 \\
\text { months: first } \\
\text { MMF over } 3 \\
\text { months then } \\
\text { CNI } 1 / 4 \text { to } 1 / 2 \\
\text { every } 3 \text { weeks }\end{array}$ & $\begin{array}{l}\text { LFT } \\
\text { Biopsy }\end{array}$ & $48.9 / \mathrm{NR} \pm 7.7$ & 98 \\
\hline $\begin{array}{r}\text { García de la } \\
\text { Garza } 2013 \\
\text { (Spain) }\end{array}$ & "Pamplona" & Adult & $\begin{array}{l}\text { Monocenter } \\
\text { Prospective }\end{array}$ & No & DD & $N R / 57$ & $\begin{array}{l}\text { non- } \\
\text { autoimmune }\end{array}$ & $\begin{array}{l}<20 \% \\
\text { replicative } \\
\text { viral } \\
\text { disease }\end{array}$ & $\begin{array}{l}\text { MMF } \\
\text { CSA } \\
\text { TAC } \\
\text { SRL }\end{array}$ & elective & Yes & $\begin{array}{l}\text { monthly } \\
\text { reductions ( } 6 \text { - } \\
10 \text { fractions) }\end{array}$ & LFT & $\begin{array}{l}\text { NR/14 (IQR 9- } \\
23 \text { ) [OT] }\end{array}$ & 24 \\
\hline $\begin{array}{r}\text { Waki } 2013 \\
\text { (Japan) }\end{array}$ & "Tochigi" & Pediatric & $\begin{array}{l}\text { Monocenter } \\
\text { Retrospective }\end{array}$ & No & LD & $\begin{array}{l}2.2 / \mathrm{NR} \pm 2.7 \\
{[\mathrm{OT}]} \\
4.3 / \mathrm{NR} \pm 4.6 \\
{[\text { nonOT] }}\end{array}$ & NR & NR & $\begin{array}{l}\mathrm{TAC}+\mathrm{CS} \\
\mathrm{CSA}+\mathrm{CS}\end{array}$ & elective & NR & NR & $\begin{array}{l}\text { LFT } \\
\text { Biopsy }\end{array}$ & NR & 40 \\
\hline $\begin{array}{l}\text { Lin } 2015 \\
\text { (Taiwan) }\end{array}$ & "Taipei" & Pediatric & $\begin{array}{l}\text { Monocenter } \\
\text { Prospective }\end{array}$ & No & both & $4.0 / \mathrm{NR} \pm 4.8$ & $\begin{array}{l}\text { non- } \\
\text { autoimmune }\end{array}$ & $\begin{array}{l}<20 \% \\
\text { replicative } \\
\text { viral } \\
\text { disease }\end{array}$ & $\begin{array}{l}\text { TAC } \\
\text { Other }\end{array}$ & elective & Yes & $\begin{array}{l}\text { stepwise } \\
\text { reduced by half } \\
\text { in 4-wk } \\
\text { intervals }\end{array}$ & $\begin{array}{l}\text { LFT } \\
\text { Biopsy }\end{array}$ & $41 / \mathrm{NR} \pm 6.0$ & 15 \\
\hline $\begin{array}{r}\text { García de la } \\
\text { Garza } 2015 \\
\text { (Spain) }\end{array}$ & "Pamplona" & Adult & $\begin{array}{l}\text { Monocenter } \\
\text { Prospective }\end{array}$ & No & DD & $N R / 57$ & $\begin{array}{l}\text { non- } \\
\text { autoimmune }\end{array}$ & $\mathrm{NR}$ & $\begin{array}{l}\text { MMF } \\
\text { TAC }\end{array}$ & elective & Yes & $\begin{array}{l}\text { monthly } \\
\text { reductions ( } 6 \text { - } \\
10 \text { fractions) }\end{array}$ & LFT & $\begin{array}{l}\text { NR/43.5 (31- } \\
60)[O T]\end{array}$ & 24 \\
\hline $\begin{array}{r}\text { Bonaccorsi- } \\
\text { Riani } 2016 \\
\text { (Spain, Italy, } \\
\text { Belgium) }\end{array}$ & $\begin{array}{l}\text { "Barcelona/R } \\
\text { ome/Leuven" } \\
\text { NCT00647283 }\end{array}$ & Adult & $\begin{array}{l}\text { Multicenter } \\
\text { Prospective }\end{array}$ & No & NR & NR & $\begin{array}{l}\text { non- } \\
\text { autoimmune }\end{array}$ & $\begin{array}{l}<20 \% \\
\text { replicative } \\
\text { viral } \\
\text { disease }\end{array}$ & $\begin{array}{l}\text { TAC } \\
\text { CSA } \\
\text { MMF } \\
\text { AZA }\end{array}$ & elective & Yes & $\begin{array}{l}\text { doses gradually } \\
\text { decreased by } \\
1 / 4 \text { to } 1 / 2\end{array}$ & $\begin{array}{l}\text { LFT } \\
\text { Biopsy }\end{array}$ & NR & 68 \\
\hline
\end{tabular}




\begin{tabular}{|c|c|c|c|c|c|c|c|c|c|c|c|c|c|c|c|}
\hline & & & & & & & & & Other & & & $\begin{array}{l}\text { within } 3 \mathrm{wk} \\
\text { over 6-9 mo }\end{array}$ & & & \\
\hline $\begin{array}{l}\text { Nafady-Hego } \\
2016 \text { (Japan) }\end{array}$ & "Kyoto" & Pediatric & $\begin{array}{l}\text { Monocenter } \\
\text { Retrospective }\end{array}$ & Yes & LD & $\begin{array}{l}{[17 / \mathrm{NR}(7-} \\
28)][\mathrm{OT}] \\
{[11 / \mathrm{NR}(5-} \\
22)][\text { nonOT] }\end{array}$ & $\begin{array}{l}\text { non- } \\
\text { autoimmune }\end{array}$ & $\begin{array}{l}\text { no patients } \\
\text { with viral } \\
\text { liver } \\
\text { disease } \\
\text { etiology }\end{array}$ & $\begin{array}{l}\text { TAC } \\
\text { TAC+CS } \\
\text { Other }\end{array}$ & $\begin{array}{l}\text { elective } \\
\text { non- } \\
\text { elective }\end{array}$ & NR & NR & & $\begin{array}{l}88 / \mathrm{NR} \pm 34 \\
{[\mathrm{OT}]}\end{array}$ & 43 \\
\hline $\begin{array}{l}\text { Revilla-Nuin } \\
2017 \text { (Spain) }\end{array}$ & $\begin{array}{l}\text { "Murcia 1-3 \& } \\
\text { Pamplona" }\end{array}$ & Adult & $\begin{array}{l}\text { Multicenter } \\
\text { Mixed }\end{array}$ & No & NR & $\begin{array}{l}53.6 / 54(40- \\
65)[\mathrm{OT}] \\
54.2 / 57(32- \\
66) \text { [nonOT] }\end{array}$ & $\begin{array}{l}<20 \% \\
\text { autoimmune }\end{array}$ & $\begin{array}{l}<20 \% \\
\text { replicative } \\
\text { viral } \\
\text { disease }^{4}\end{array}$ & $\begin{array}{l}\text { CsA } \\
\text { TAC } \\
\text { MMF } \\
\text { SRL } \\
\text { EVL } \\
\text { Other }\end{array}$ & elective & Yes & $\begin{array}{l}\geq 10 \% \\
\text { decrements at } \\
1 \text { mo intervals }\end{array}$ & $\begin{array}{l}\text { LFT } \\
\text { Biopsy }\end{array}$ & $\begin{array}{l}54 / 60(12- \\
144)[O T] \\
47 / 36(1-180) \\
\text { [nonOT] }\end{array}$ & 47 \\
\hline $\begin{array}{l}\text { Baroja-Mazo } \\
2019 \text { (Spain) }\end{array}$ & $\begin{array}{l}\text { "Murcia 3" } \\
\text { ISRCTN15775 } \\
356\end{array}$ & Adult & $\begin{array}{l}\text { Monocenter } \\
\text { Prospective }\end{array}$ & No & NR & $\begin{array}{l}58.6 / 59 \text { (52- } \\
65)[\mathrm{OT}] \\
62.4 / 63.5 \\
(56-71) \\
\text { [nonOT] }\end{array}$ & $\begin{array}{l}\text { non- } \\
\text { autoimmune }\end{array}$ & $\begin{array}{l}<20 \% \\
\text { replicative } \\
\text { viral } \\
\text { disease }^{4}\end{array}$ & $\begin{array}{l}\text { CsA } \\
\text { TAC } \\
\text { TAC+CS } \\
\text { Other }\end{array}$ & elective & Yes & $\begin{array}{l}\geq 10 \% \\
\text { decrements at } \\
1 \text { mo intervals }\end{array}$ & $\begin{array}{l}\text { LFT } \\
\text { Biopsy }\end{array}$ & NR & 17 \\
\hline $\begin{array}{r}\text { Perez-Sanz } \\
2019 \text { (Spain) }\end{array}$ & $\begin{array}{l}\text { "Murcia 3" } \\
\text { ISRCTN15775 } \\
356\end{array}$ & Adult & $\begin{array}{l}\text { Monocenter } \\
\text { Prospective }\end{array}$ & No & NR & $\begin{array}{l}58.6 / 59(52- \\
65)[\mathrm{OT}] \\
62.4 / 63.5 \\
(56-71) \\
\text { [nonOT] }\end{array}$ & $\begin{array}{l}\text { non- } \\
\text { autoimmune }\end{array}$ & $\begin{array}{l}<20 \% \\
\text { replicative } \\
\text { viral } \\
\text { disease }^{4}\end{array}$ & $\begin{array}{l}\text { CsA } \\
\text { TAC } \\
\text { TAC+CS } \\
\text { Other }\end{array}$ & elective & Yes & $\begin{array}{l}\geq 10 \% \\
\text { decrements at } \\
1 \text { mo intervals }\end{array}$ & $\begin{array}{l}\text { LFT } \\
\text { Biopsy }\end{array}$ & NR & 17 \\
\hline $\begin{array}{r}\text { Levitsky } 2020 \\
\text { (USA) }\end{array}$ & $\begin{array}{l}\text { "Chicago" } \\
\text { NCT02062944 }\end{array}$ & Adult & $\begin{array}{l}\text { Monocenter } \\
\text { Prospective }\end{array}$ & No & both & $\begin{array}{l}{[63.7(47-76)} \\
{[O T]} \\
62.7(44-67) \\
{[\text { nonOT]] }}\end{array}$ & $\begin{array}{l}\text { non- } \\
\text { autoimmune }\end{array}$ & $\begin{array}{l}<20 \% \\
\text { replicative } \\
\text { viral } \\
\text { disease }\end{array}$ & SRL & elective & Yes & $\begin{array}{l}\text { slowly over 3-6 } \\
\text { months } \\
\text { (reduced every } \\
\text { month by } 50 \% \\
\text { of total dose } \\
\text { until } 0.5 \mathrm{mg} \\
\text { daily for one } \\
\text { month, then } \\
\text { each month } 0.5 \\
\text { mg every other } \\
\text { day, twice wkly, } \\
\text { finally once } \\
\text { wkly) }\end{array}$ & $\begin{array}{l}\text { LFT } \\
\text { Biopsy }\end{array}$ & NR & 15 \\
\hline $\begin{array}{r}\text { Feng } 2020 \\
\text { (USA, Canada) }\end{array}$ & $\begin{array}{l}\text { "iWITH" } \\
\text { NCTO1638559 }\end{array}$ & Pediatric & $\begin{array}{l}\text { Multicenter } \\
\text { Prospective }\end{array}$ & No & both & $\begin{array}{l}\text { NR/1 IQR 1-2 } \\
\text { [OT] } \\
\text { NR/1 IQR 1-3 } \\
\text { [nonOT] }\end{array}$ & $\begin{array}{l}\text { non- } \\
\text { autoimmune }\end{array}$ & $\begin{array}{l}\text { no patients } \\
\text { with viral } \\
\text { liver } \\
\text { disease } \\
\text { etiology }\end{array}$ & $\begin{array}{l}\text { TAC } \\
\text { CSA }\end{array}$ & elective & Yes & $\begin{array}{l}\text { reduced in } 7 \\
\text { steps of } 4 \text { or } 6 \\
\text { wk duration ( } 36 \\
\text { - } 48 \text { wk total) }\end{array}$ & $\begin{array}{l}\text { LFT } \\
\text { Biopsy }\end{array}$ & « yr» & 88 \\
\hline
\end{tabular}

${ }^{1}$ This Barcelona study is attached to the multicenter "Barcelona/Rome/Leuven" study due to cohort overlap.

${ }^{2}$ [age at study] if age at LT not reported

${ }^{3}$ corrected months to years

${ }^{4}$ Alberto Baroja-Mazo, personal communication 20200706

AZA, azathioprine; CNI, calcineurin inhibitor; CS, corticosteroid; CSA, cyclosporine A; DD, deceased donor; EVL, everolimus; FU, follow-up; IQR, interquartile range; IS, immunosuppression; ISW, immunosuppression withdrawal; LD, living donor; LFT, liver function tests; LT, liver transplantation; MMF, mycophenolate mofetil; mo, months; NR, not reported; OT, operational tolerance; SRL, sirolimus; TAC, tacrolimus; wk, weeks; wkly, weekly; yr, years 


\section{Table S4: Included conference abstracts ${ }^{\S}$}

\begin{tabular}{|c|c|c|c|}
\hline $\begin{array}{l}\text { First Author } \\
\text { Year }\end{array}$ & Journal & Title & Study Population \\
\hline $\begin{array}{l}\text { Feng } \\
2014^{47}\end{array}$ & Transplantation & $\begin{array}{l}\text { High percentage of PD-1+CD4+ T cells in a subset of tolerant pediatric } \\
\text { liver recipients }\end{array}$ & pediatric \\
\hline $\begin{array}{l}\text { Ramaswami } \\
2014^{42}\end{array}$ & Transplantation & $\begin{array}{l}\text { Operationally tolerant pediatric liver transplant recipients exhibit } \\
\text { features of immune activation and exhaustion }\end{array}$ & pediatric \\
\hline $\begin{array}{l}\text { Ramaswami } \\
2014^{44}\end{array}$ & $\begin{array}{l}\text { Journal of } \\
\text { Immunology }\end{array}$ & $\begin{array}{l}\text { Pediatric liver transplant recipients with operational tolerance exhibit } \\
\text { features of immune activation and exhaustion }\end{array}$ & pediatric \\
\hline $\begin{array}{l}\text { Danger } \\
2015^{43}\end{array}$ & $\begin{array}{l}\text { American Journal of } \\
\text { Transplantation }\end{array}$ & $\begin{array}{l}\text { Hepatocyte-related miR-193a-3p is over-expressed in allograft biopsy } \\
\text { from liver tolerant recipients }\end{array}$ & adult \\
\hline $\begin{array}{l}\text { Ramaswami } \\
2015^{46}\end{array}$ & Transplantation & $\begin{array}{l}\text { Pediatric liver transplant recipients with operational tolerance exhibit } \\
\text { features of immune activation and exhaustion }\end{array}$ & pediatric \\
\hline $\begin{array}{l}\text { Burrell } \\
2017^{45}\end{array}$ & $\begin{array}{l}\text { American Journal of } \\
\text { Transplantation }\end{array}$ & $\begin{array}{l}\text { Tolerant pediatric liver transplant (LT) recipients (TOL) from WISP-R } \\
\text { (NCT00320606) split into two phenotypically distinct groups by PD1 } \\
\text { expression on CD4+ T cells at baseline, prior to immunosuppression } \\
\text { withdrawal (ISW) }\end{array}$ & pediatric \\
\hline $\begin{array}{l}\text { Chandran } \\
2017^{38}\end{array}$ & $\begin{array}{l}\text { American Journal of } \\
\text { Transplantation }\end{array}$ & $\begin{array}{l}\text { Subclinical histologic findings are observed in } 25 \% \text { of stable adult liver } \\
\text { transplant recipients (ALTRs) screened for immunosuppression } \\
\text { withdrawal (ISW): OPTIMAL (NCT02533180) }\end{array}$ & adult \\
\hline $\begin{array}{l}\text { Chruscinski } \\
2017^{39}\end{array}$ & $\begin{array}{l}\text { Transplant } \\
\text { International }\end{array}$ & $\begin{array}{l}\text { Results of litmus (NCT 02541916): The liver immune tolerance } \\
\text { biomarker utilization study }\end{array}$ & adult \\
\hline $\begin{array}{l}\text { Chruscinski } \\
2018^{40}\end{array}$ & $\begin{array}{l}\text { American Journal of } \\
\text { Transplantation }\end{array}$ & $\begin{array}{l}\text { Results of litmus (nct 02541916): The liver immune tolerance bio marker } \\
\text { utilization study }\end{array}$ & adult \\
\hline $\begin{array}{l}\text { Chruscinski } \\
2018^{48}\end{array}$ & Transplantation & $\begin{array}{l}\text { Results of LITMUS (NCT 02541916): The liver immune tolerance bio } \\
\text { marker utilization study }\end{array}$ & adult \\
\hline $\begin{array}{l}\mathrm{Gu} \\
2018^{36}\end{array}$ & Transplantation & $\begin{array}{l}\text { Liver transplant tolerance induced by severe or consistent infection } \\
\text { revealed the association with T cell exhaustion }\end{array}$ & pediatric \\
\hline $\begin{array}{l}\text { Sanchez- } \\
\text { Fueyo } \\
2018^{37}\end{array}$ & $\begin{array}{l}\text { Journal of } \\
\text { Hepatology }\end{array}$ & $\begin{array}{l}\text { Prevalence of subclinical histological lesions and tolerance biomarkers in } \\
\text { long-term adult liver transplant recipients considered for } \\
\text { immunosuppression withdrawal }\end{array}$ & adult \\
\hline $\begin{array}{l}\text { Vionnet } \\
2019^{41}\end{array}$ & $\begin{array}{l}\text { American Journal of } \\
\text { Transplantation }\end{array}$ & $\begin{array}{l}\text { Identification of adult liver transplant recipients eligible to participate in } \\
\text { an immunosuppression withdrawal trial employing non-invasive } \\
\text { assessments of allograft status }\end{array}$ & adult \\
\hline
\end{tabular}

$\S$ The data issued from conference abstracts is not peer-reviewed. 


\section{Table S5: Included registered trials}

\begin{tabular}{|c|c|c|c|}
\hline $\begin{array}{l}\text { Principal } \\
\text { Investigator } \\
\text { Year }\end{array}$ & $\begin{array}{l}\text { Trial ID } \\
\text { Trial Name }\end{array}$ & Title & $\begin{array}{l}\text { Study } \\
\text { population }\end{array}$ \\
\hline $\begin{array}{l}\text { Sanchez- } \\
\text { Fueyo } \\
2011 \\
\end{array}$ & NCT01445236 & $\begin{array}{l}\text { Pilot Study of Immunosuppression Drug Weaning in Liver } \\
\text { Recipients Exhibiting Biomarkers of High Likelihood of Tolerance }\end{array}$ & adult \\
\hline $\begin{array}{l}\text { Sanchez- } \\
\text { Fueyo } \\
2015\end{array}$ & $\begin{array}{l}\text { NCT02498977 } \\
\text { LIFT }\end{array}$ & Liver Immunosuppression Free Trial & adult \\
\hline $\begin{array}{l}\text { Markmann } \\
2015\end{array}$ & $\begin{array}{l}\text { NCT02533180 } \\
\text { OPTIMAL }\end{array}$ & $\begin{array}{l}\text { Evaluation of Donor Specific Immune Senescence and Exhaustion } \\
\text { as Biomarkers of Tolerance Post Liver Transplantation }\end{array}$ & adult \\
\hline $\begin{array}{l}\text { Levy } \\
2015\end{array}$ & $\begin{array}{l}\text { NCT02541916 } \\
\text { LITMUS }\end{array}$ & Liver Immune Tolerance Marker Utilization Study & adult \\
\hline $\begin{array}{l}\text { Chandran } \\
2016\end{array}$ & $\begin{array}{l}\text { NCT02743793 } \\
\text { ALLTOL }\end{array}$ & A Cohort Study of Operationally Tolerant Allograft Recipients & adult \\
\hline
\end{tabular}




\begin{tabular}{|c|c|c|c|c|c|c|c|c|c|c|c|c|c|c|}
\hline 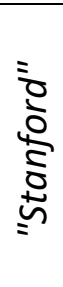 & $\begin{array}{l}\text { Talisetti } \\
2010\end{array}$ & $\begin{array}{l}18 / 62 \\
(29 \%)\end{array}$ & 0 & & & & & $\begin{array}{l}\text { recipient } \\
\text { age at LT }\end{array}$ & $\begin{array}{l}\text { mean (range) } \\
{[y r]}\end{array}$ & $\begin{array}{l}1.4(0.3 \\
-4.9)\end{array}$ & $\begin{array}{l}3.4(0.3 \\
-16)\end{array}$ & & & $\begin{array}{l}\text { sex } \\
\text { liver disease etiology } \\
\text { ABO compatibility of graft } \\
\text { Donor age } \\
\text { sex mismatch } D / R\end{array}$ \\
\hline \multirow{7}{*}{ 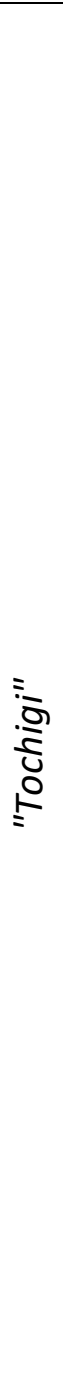 } & Waki 2011 & $\begin{array}{l}18 / 52 \\
(35 \%)\end{array}$ & 1 & & & & & sex & $\%$ male & 11.1 & 48.5 & & & $\begin{array}{l}\text { lymphocytotoxic } \\
\text { crossmatch } \\
\text { HLA mismatch } \\
\text { recipient age at LT } \\
\text { liver disease etiology } \\
\text { history of rejection } \\
\text { Donor age } \\
\text { Postoperative } \\
\text { complications (biliary, } \\
\text { vascular) } \\
\text { Cold or warm ischemia time } \\
\text { PELD score }\end{array}$ \\
\hline & Waki 2013 & $\begin{array}{l}17 / 40 \\
(43 \%)\end{array}$ & 0 & $\begin{array}{l}\text { Anti-HLA } \\
\text { antibodies } \\
\text { Anti-HLA } \\
\text { antibodies }\end{array}$ & $\begin{array}{l}\text { post-LT } \\
\text { class I; } \% \\
\text { patients } \\
\text { post-LT } \\
\text { class II; } \% \\
\text { patients }\end{array}$ & 27 & 67 & sex & $\%$ male & 11.8 & 52.2 & $\begin{array}{l}\begin{array}{l}\text { Anti-HLA } \\
\text { antibodies }\end{array} \\
\text { Anti-HLA } \\
\text { antibodies }\end{array}$ & $\begin{array}{l}\text { pre-LT } \\
\text { post-LT } \\
\text { HLA-A }\end{array}$ & liver disease etiology \\
\hline & & & & $\begin{array}{l}\text { Anti-HLA } \\
\text { antibodies }\end{array}$ & $\begin{array}{l}\text { post-LT } \\
\text { HLA-B; \% } \\
\text { patients }\end{array}$ & 20 & 67 & & & & & $\begin{array}{l}\text { Anti-HLA } \\
\text { antibodies }\end{array}$ & $\begin{array}{l}\text { post-LT } \\
\text { HLA-DP }\end{array}$ & HLA mismatch \\
\hline & & & & $\begin{array}{l}\text { Anti-HLA } \\
\text { antibodies }\end{array}$ & $\begin{array}{l}\text { post-LT } \\
\text { HLA-C; \% } \\
\text { patients }\end{array}$ & 33 & 76 & & & & & & & $\begin{array}{l}\text { Lymphocytotoxic } \\
\text { crossmatch }\end{array}$ \\
\hline & & & & $\begin{array}{l}\text { Anti-HLA } \\
\text { antibodies }\end{array}$ & $\begin{array}{l}\text { post-LT } \\
\text { HLA-DQ; \% } \\
\text { patients }\end{array}$ & 7 & 43 & & & & & & & time from LT to ISW \\
\hline & & & & $\begin{array}{l}\text { Anti-HLA } \\
\text { antibodies }\end{array}$ & $\begin{array}{l}\text { post-LT } \\
\text { HLA-DR; \% } \\
\text { patients }\end{array}$ & 20 & 57 & & & & & & & Donor age \\
\hline & & & & & & & & & & & & & & $\begin{array}{l}\text { Cold or warm ischemia time } \\
\text { PELD score }\end{array}$ \\
\hline
\end{tabular}




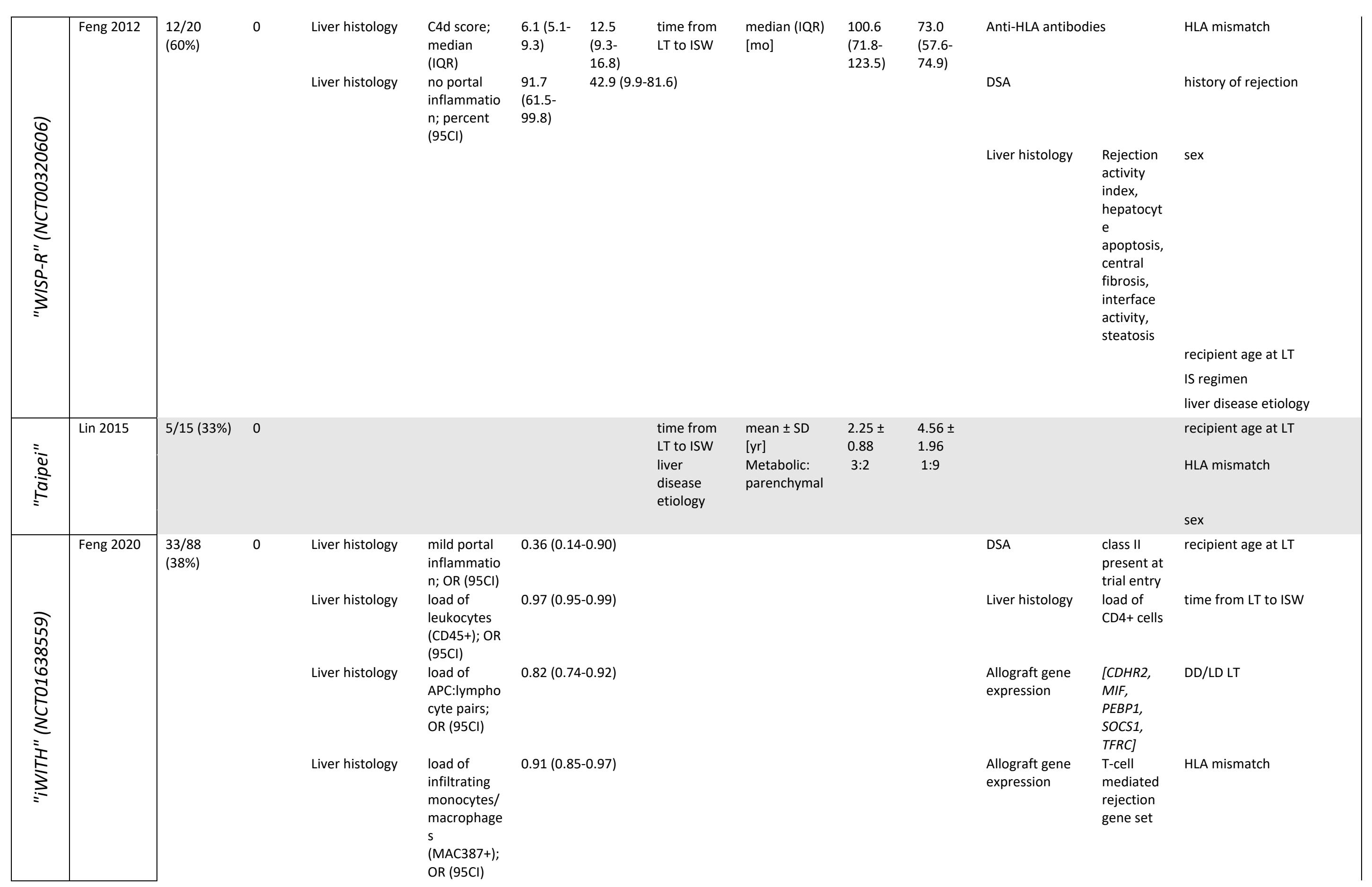




\section{only by univariate analysis}

ALT, alanine aminotransferase; APC, antigen-presenting cell; AST, aspartate aminotransferase; D, donor; d, days; DD, deceased donor; DSA, donor-specific antibodies; FU, follow-up; LmIGGT, gamma glutamyltransferase; HLA, human leukocyte antigen; IQR, interquartile range; IS, immunosuppression; ISW, immunosuppression withdrawal; L, liter; LD, living donor; LT, liver

transplantation; ml, milliliters; mo, months; ng, nanograms; OR, odds ratio; PELD, Pediatric End-stage Liver Disease; R, recipient; SD, standard deviation; U, units; WBC, white blood cells; wk, weeks; yr, years; $95 \mathrm{Cl}, 95 \%$ confidence interval 
Table S7: Summary of Results from Adult Studies

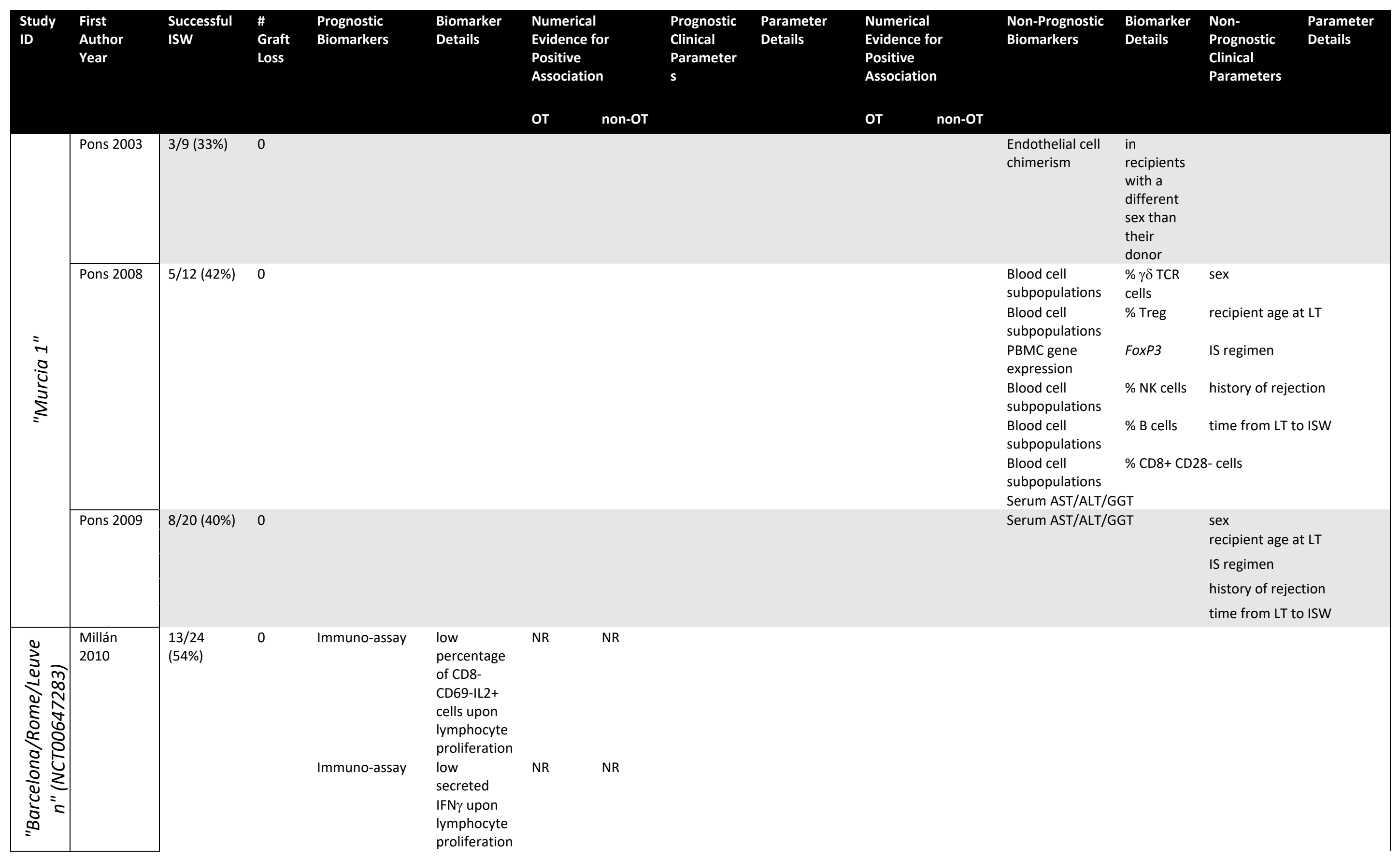




\begin{tabular}{|c|c|c|c|c|c|c|c|c|c|c|c|c|c|}
\hline \multirow[t]{5}{*}{$\begin{array}{l}\text { Bohne } \\
2012\end{array}$} & \multirow[t]{5}{*}{$\begin{array}{l}33 / 75 \\
(44 \%)\end{array}$} & \multirow[t]{5}{*}{0} & $\begin{array}{l}\text { Allograft gene } \\
\text { expression }\end{array}$ & $\begin{array}{l}\text { [CDHR2, } \\
\text { MIF, PEBP1, } \\
\text { SOCS1, } \\
\text { TFRC]; OR } \\
\text { (95CI) } \\
\text { (and } 4 \\
\text { additional } \\
\text { gene } \\
\text { signatures) }\end{array}$ & \multicolumn{2}{|c|}{$\begin{array}{l}49 \text { (7-343) } \\
(89 \% \text { SN, 86\% SP, } \\
80 \% \text { PPV, 92\% NPV, } \\
13 \% \text { ER) and ( } 80 \% \\
\text { SN, 100\% SP, 100\% } \\
\text { PPV, 85\% NPV, } \\
9.5 \% \text { ER) } \\
\text { AUC } 0.83\end{array}$} & $\begin{array}{l}\text { time from } \\
\text { LT to ISW }\end{array}$ & $\begin{array}{l}\text { median } \\
\text { (range) [mo] }\end{array}$ & $\begin{array}{l}143(52- \\
212)\end{array}$ & $\begin{array}{l}86(36- \\
215)\end{array}$ & $\begin{array}{l}\text { Blood cell } \\
\text { subpopulations }\end{array}$ & $\%$ Treg & sex \\
\hline & & & $\begin{array}{l}\text { PBMC gene } \\
\text { expression }\end{array}$ & $\begin{array}{l}\text { [NCR1, } \\
\text { PDGFRB, } \\
\text { PSMD14] or } \\
\text { [SLAMF7, } \\
\text { KLRF1, } \\
\text { CLIC3, } \\
\text { PSMD14, } \\
\text { ALG8, } \\
\text { CX3CR1] }\end{array}$ & \multicolumn{2}{|c|}{$\begin{array}{l}\text { AUC } 0.76 \\
\text { AUC } 0.71\end{array}$} & $\begin{array}{l}\text { recipient } \\
\text { age at } L T\end{array}$ & $\begin{array}{l}\text { median } \\
\text { (range) [yr] }\end{array}$ & $\begin{array}{l}62(27- \\
73)\end{array}$ & $\begin{array}{l}51(25- \\
71)\end{array}$ & \multirow{4}{*}{\multicolumn{2}{|c|}{ Liver histology }} & \\
\hline & & & $\begin{array}{l}\text { Iron status } \\
\text { Iron status } \\
\text { Liver histology }\end{array}$ & $\begin{array}{l}\text { serum } \\
\text { ferritin; } \\
\text { mean } \\
\text { (range) } \\
\text { serum } \\
\text { hepcidin } 25 \\
\text { hepatocytic } \\
\text { iron }\end{array}$ & $\begin{array}{l}185.5 \\
(26- \\
864)\end{array}$ & $\begin{array}{l}73.5(3- \\
304)\end{array}$ & IS regimen & $\begin{array}{l}\% \text { patients } \\
\text { with CNI }\end{array}$ & 66.7 & 91.3 & & & \\
\hline & & & $\begin{array}{l}\text { Blood cell } \\
\text { subpopulations }\end{array}$ & $\%$ NK cells & & & & & & & & & \\
\hline & & & $\begin{array}{l}\text { Blood cell } \\
\text { subpopulations }\end{array}$ & $\begin{array}{l}\% \gamma \delta \text { TCR } \\
\text { cells }\end{array}$ & & & & & & & & & \\
\hline \multirow[t]{6}{*}{$\begin{array}{l}\text { Benítez } \\
2013\end{array}$} & \multirow[t]{6}{*}{$\begin{array}{l}41 / 98 \\
(42 \%)\end{array}$} & \multirow[t]{6}{*}{0} & \multirow[t]{6}{*}{ Serum GGT } & \multirow[t]{6}{*}{$\begin{array}{l}\text { mean } \pm S D \\
{[U / L]}\end{array}$} & \multirow[t]{6}{*}{$50 \pm 70$} & \multirow[t]{6}{*}{$27 \pm 28$} & $\begin{array}{l}\text { recipient } \\
\text { age at } L T\end{array}$ & $\begin{array}{l}\text { mean } \pm S D \\
{[y r]}\end{array}$ & $50 \pm 10$ & & $\begin{array}{l}\text { Anti-HLA } \\
\text { antibodies }\end{array}$ & $\begin{array}{l}\text { class I+ } \\
\text { class II }\end{array}$ & \\
\hline & & & & & & & sex & $\%$ male & 83 & 58 & $\begin{array}{l}\text { Serum total } \\
\text { bilirubin }\end{array}$ & & history of rejection \\
\hline & & & & & & & $\begin{array}{l}\text { time from } \\
\text { LT to ISW }\end{array}$ & $\begin{array}{l}\text { mean } \pm S D \\
{[\mathrm{mo}]}\end{array}$ & $131 \pm 47$ & $83 \pm 40$ & \multirow{4}{*}{\multicolumn{2}{|c|}{ Serum AST/ALT/GGT }} & HLA mismatch \\
\hline & & & & & & & IS regimen & $\begin{array}{l}\% \text { patients } \\
\text { with } \mathrm{CNI}\end{array}$ & 56 & 79 & & & Donor age \\
\hline & & & & & & & & & & & & & $\begin{array}{ll}\text { IS regimen } & \begin{array}{l}\text { trough } \\
\text { levels }\end{array}\end{array}$ \\
\hline & & & & & & & & & & & & & $\begin{array}{l}\text { Renal function (creatinine, } \\
\text { GFR) }\end{array}$ \\
\hline $\begin{array}{l}\text { Bonaccorsi } \\
\text {-Riani } 2016\end{array}$ & $\begin{array}{l}25 / 68 \\
(37 \%)\end{array}$ & 0 & $\begin{array}{l}\text { PBMC gene } \\
\text { expression }\end{array}$ & FoxP3 & up & down & & & & & $\begin{array}{l}\text { PBMC gene } \\
\text { expression }\end{array}$ & $\begin{array}{l}\text { CD3 } \\
\text { MAN1A1 } \\
\text { PRF1 } \\
\text { Toag-1 }\end{array}$ & \\
\hline
\end{tabular}




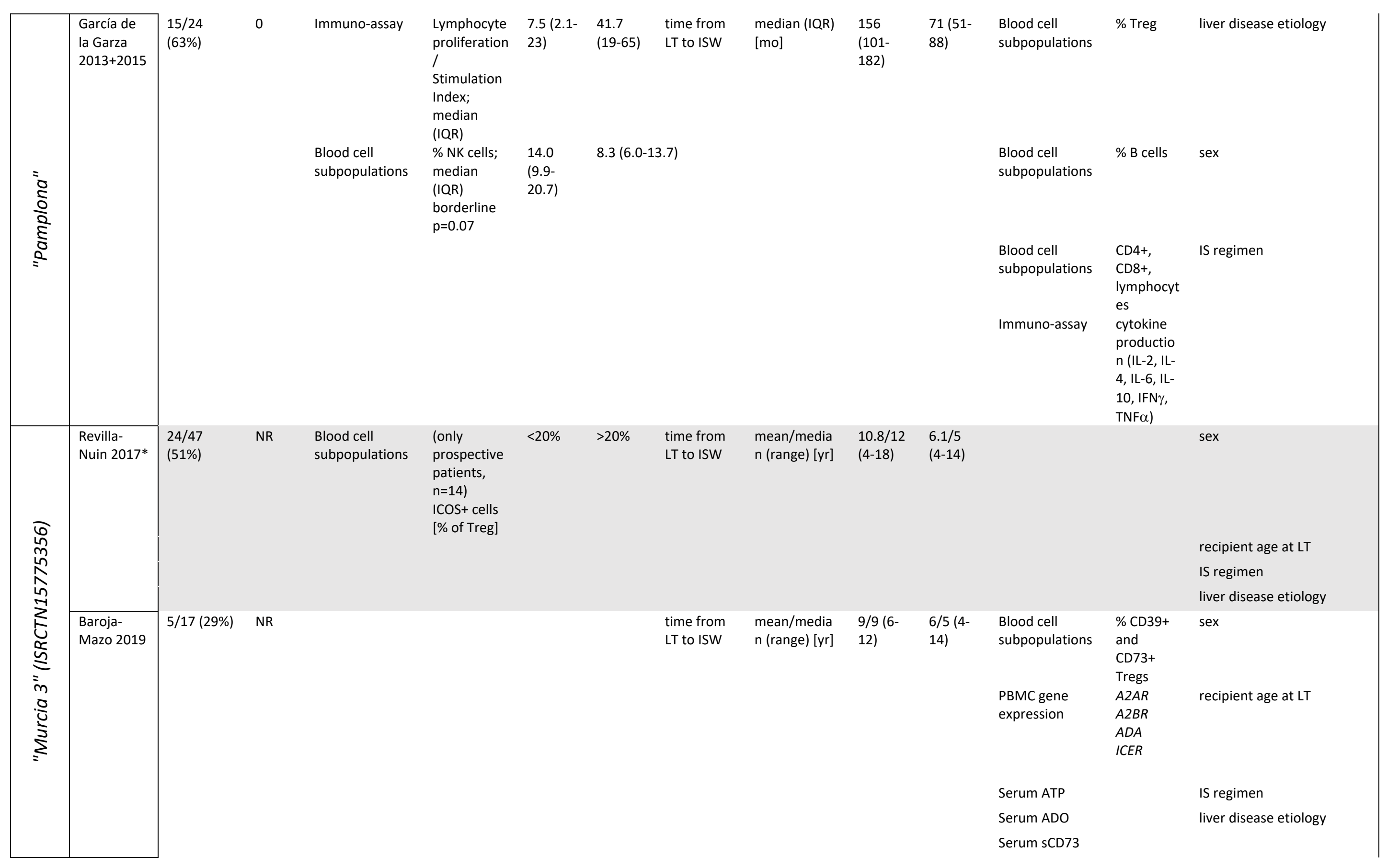




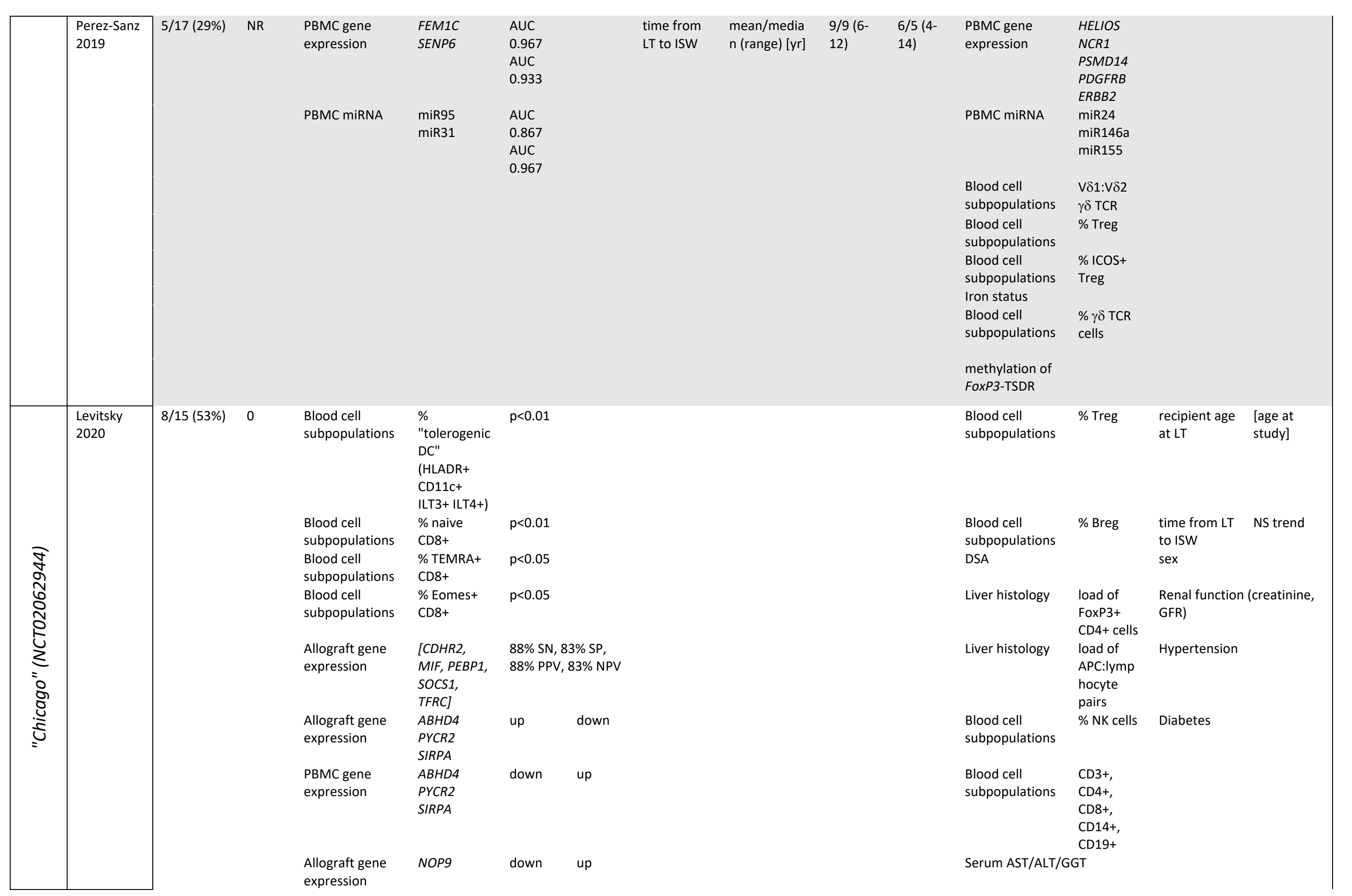




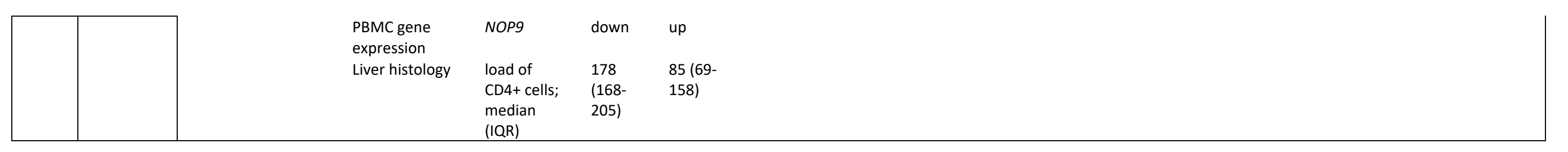

* $\quad$ This report also includes patients from other cohorts ("Pamplona", "Murcia 1", "Murcia 2")

ADO, adenosine; ALT, alanine aminotransferase; APC, antigen-presenting cell; AST, aspartate aminotransferase; ATP, adenosine triphosphate; AUC, area under the curve; Breg, regulatory B-cell;

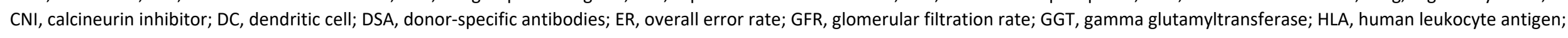
IQR, interquartile range; IS, immunosuppression; ISW, immunosuppression withdrawal; L, liter; LT, liver transplantation; mo, months; PBMCNK, natural killer; NPV, negative predictive value; NR, not reported; NS, non-significant; OR, odds ratio; PBMC, peripheral blood mononuclear cells; PPV, positive predictive value; sCD73, soluble CD73; SD, standard deviation; SN, sensitivity; SP, specificity; TCR, T-cell receptor; Treg, regulatory T-cell; TSDR, T-cell-specific demethylated region; U, units; yr, years; $95 \mathrm{Cl}, 95 \%$ confidence interval 